\title{
C-type lectin-like receptor 2 (CLEC-2)-dependent dendritic cell migration is controlled by tetraspanin CD37
}

\author{
Charlotte M. de Winde ${ }^{1,2}$, Alexandra L. Matthews ${ }^{3, *}$, Sjoerd van Deventer ${ }^{1, *}$, Alie van der Schaaf ${ }^{1}$, \\ Neil D. Tomlinson ${ }^{4}$, Erik Jansen ${ }^{1}$, Johannes A. Eble ${ }^{5}$, Bernhard Nieswandt ${ }^{6}$, Helen M. McGettrick ${ }^{7}$, \\ Carl G. Figdor ${ }^{1}$, Michael G. Tomlinson ${ }^{3,8}$, Sophie E. Acton ${ }^{2}$ and Annemiek B. van Spriel ${ }^{1, \pm}$
}

\begin{abstract}
Cell migration is central to evoking a potent immune response. Dendritic cell (DC) migration to lymph nodes is dependent on the interaction of C-type lectin-like receptor 2 (CLEC-2; encoded by the gene Clec1b), expressed by DCs, with podoplanin, expressed by lymph node stromal cells, although the underlying molecular mechanisms remain elusive. Here, we show that CLEC-2-dependent DC migration is controlled by tetraspanin CD37, a membrane-organizing protein. We identified a specific interaction between CLEC-2 and CD37, and myeloid cells

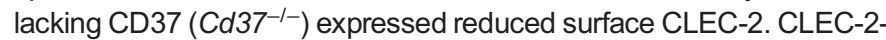
expressing $\mathrm{Cd} 37^{-1-} \mathrm{DCs}$ showed impaired adhesion, migration velocity and displacement on lymph node stromal cells. Moreover, $\mathrm{Cd} 37^{-1-} \mathrm{DCs}$ failed to form actin protrusions in a 3D collagen matrix upon podoplanininduced CLEC-2 stimulation, phenocopying CLEC-2-deficient DCs. Microcontact printing experiments revealed that CD37 is required for CLEC-2 recruitment in the membrane to its ligand podoplanin. Finally, $\mathrm{Cd} 37^{-1-}$ DCs failed to inhibit actomyosin contractility in lymph node stromal cells, thus phenocopying CLEC-2-deficient DCs. This study demonstrates that tetraspanin CD37 controls CLEC-2 membrane organization and provides new molecular insights into the mechanisms underlying CLEC-2-dependent DC migration.
\end{abstract}

This article has an associated First Person interview with the first author of the paper.

KEY WORDS: CLEC-2, Dendritic cell, Migration, Membrane organization, Tetraspanin

\section{INTRODUCTION}

Cell migration is a key process in the initiation of immune responses (Worbs et al., 2017). Upon encountering a foreign antigen, dendritic cells (DCs) migrate to secondary lymphoid organs (i.e. lymph nodes, spleen) to present antigen on major histocompatibility

\footnotetext{
${ }^{1}$ Radboud University Medical Center, Radboud Institute for Molecular Life Sciences, Department of Tumor Immunology, 6525 GA Nijmegen, The Netherlands. ${ }^{2}$ MRC Laboratory of Molecular Cell Biology, University College London, London WC1E 6BT UK. ${ }^{3}$ School of Biosciences, University of Birmingham, Birmingham B15 2TT, UK. ${ }^{4}$ Institute of Cardiovascular Sciences, University of Birmingham, Edgbaston, Birmingham B15 2TT, UK. ${ }^{5}$ Institute for Physiological Chemistry and Pathobiochemistry, D-48149 Münster, Germany. ${ }^{6}$ University Clinic of Würzburg and Rudolf Virchow Center for Experimental Biomedicine, 97070 Würzburg, Germany. ${ }^{7}$ Institute of Inflammation and Ageing, University of Birmingham, Edgbaston, Birmingham B15 2TT, UK. ${ }^{8}$ Centre of Membrane Proteins and Receptors (COMPARE), Universities of Birmingham and Nottingham, Midlands, UK. *These authors contributed equally to this work

‡Author for correspondence (annemiek.vanspriel@radboudumc.nl)
}

(D) C.M.d.W., 0000-0002-8318-4612; N.D.T., 0000-0001-7377-9789; A.B.v.S., 0000-0002-3590-2368

Received 2 January 2018; Accepted 23 August 2018 complex (MHC) and activate T and B lymphocytes. En route, DCs move along podoplanin-expressing lymph node stromal cells (LNSCs), such as lymphatic endothelial cells (LECs) and fibroblastic reticular cells (FRCs). The interaction between C-type lectin-like receptor 2 (CLEC-2) on DCs with podoplanin on LNSCs is essential for optimal DC migration to and within the lymph node (Acton et al., 2012), and inhibits FRC actomyosin contractility, resulting in lymph node expansion (Acton et al., 2014). Despite the important role of CLEC-2 in DC migration, the molecular mechanisms underlying CLEC-2-dependent cell migration have yet to be elucidated.

CLEC-2 (encoded by the gene Clec $1 b$ ) is expressed by platelets (Suzuki-Inoue et al., 2006) and myeloid immune cells, such as DCs, macrophages and neutrophils (Colonna et al., 2000; Lowe et al., 2015; Mourão-Sá et al., 2011). CLEC-2 plays a key role in fetal development of the lymphatic vasculature, as demonstrated by Cleclb-knockout mice, which are embryonically lethal (Bertozzi et al., 2010; Suzuki-Inoue et al., 2010). Besides podoplanin, the snake venom toxin rhodocytin is another ligand for CLEC-2. Both ligands initiate downstream signalling via Syk, resulting in cell activation (Fuller et al., 2007; Hughes et al., 2010; Suzuki-Inoue et al., 2006). Intracellular Syk binding requires the dimerization of CLEC-2 receptors, because each CLEC-2 receptor contains only a single tyrosine phosphorylation (YXXL) motif. This makes CLEC-2 a hemiimmunoreceptor tyrosine-based activation motif (hemITAM) C-type lectin receptor (CLR), similar to its homologous family member Dectin-1 (also known as CLEC7A), which recognizes $\beta$-glucans in fungal cell walls (Brown and Gordon, 2001; Fuller et al., 2007).

The organization of receptors in the plasma membrane of DCs plays a pivotal role in immune cell function (Zuidscherwoude et al., 2014, 2017a). For proper ligand binding and initiation of signalling, CLRs are dependent on localization into membrane microdomains, such as lipid rafts and tetraspanin-enriched microdomains (TEMs) (Figdor and van Spriel, 2009; Zuidscherwoude et al., 2014). TEMs, also referred to as the tetraspanin web, are formed by the interaction of tetraspanins, a family of four-transmembrane proteins, with each other and partner proteins (Charrin et al., 2009; Hemler, 2005; Levy and Shoham, 2005; Zimmerman et al., 2016; Zuidscherwoude et al., 2015). As such, TEMs have been implicated in fundamental cell biological functions, including proliferation, adhesion and signalling (Charrin et al., 2009; Hemler, 2005; Levy and Shoham, 2005). Earlier work indicated that CLEC-2 clustering and signalling in blood platelets is dependent on lipid rafts (Manne et al., 2015; Pollitt et al., 2010). CLEC-2 was shown to be present as single molecules or homodimers on resting platelets, and larger clusters were formed upon rhodocytin stimulation (Hughes et al., 2010; Pollitt et al., 2014), but the molecular mechanism underlying ligand-induced CLEC-2 clustering is as yet unknown. More is 
known about the organization of Dectin-1, a CLEC-2 homologous family member, on immune cells. Dectin-1 molecules need to be reorganized into a 'phagocytic cup' to bind and phagocytose particulate, and not soluble, $\beta$-glucan (Goodridge et al., 2011). Furthermore, Dectin-1 has been proposed to be present in lipid rafts (De Turris et al., 2015; Xu et al., 2009) or tetraspanin (CD63, CD37) microdomains (Mantegazza et al., 2004; Meyer-Wentrup et al., 2007; Yan et al., 2014) on myeloid cells.

Tetraspanin CD37 is exclusively expressed by immune cells, with highest expression on B lymphocytes and DCs (de Winde et al., 2015). The importance of CD37 in the immune system has been demonstrated in $\mathrm{CD} 37$-deficient mice $\left(\mathrm{Cd}_{3} 7^{-/}\right)$that have defective humoral and cellular immune responses (van Spriel et al., 2004, 2012). Interestingly, DCs that lack CD37 showed impaired spreading, adhesion and migration, leading to defective initiation of the cellular immune response (Gartlan et al., 2013; Jones et al., 2016).

Because CLEC-2 plays an important role in DC migration (Acton et al., 2012), and its homologous receptor Dectin-1 has been shown to interact with tetraspanin CD37 (Meyer-Wentrup et al., 2007), we hypothesized that CD37 might influence CLEC-2 membrane organization and thereby control DC migration. In this study, we show that CLEC-2 interacts with CD37. Moreover, CLEC-2dependent actin protrusion formation by DCs and recruitment of CLEC-2 expressed on RAW264.7 (RAW) macrophages to podoplanin is dependent on CD37 expression. Our data also indicate that CD37 is important for reciprocal signalling upon the interaction between CLEC-2 and podoplanin, resulting in loss of actomyosin contractility in FRCs. These results provide evidence that tetraspanin $\mathrm{CD} 37$ is required for CLEC-2 recruitment to the plasma membrane in response to podoplanin, and, as such, plays an important role in CLEC-2-dependent DC migration.

\section{RESULTS}

\section{CLEC-2 specifically interacts with tetraspanin CD37}

To investigate whether CLEC-2 interacts with tetraspanins, we performed co-immunoprecipitation experiments in lysates of human embryonic kidney (HEK-293T) cells transfected with human (h)CLEC-2-MYC with or without a range of FLAG-tagged human tetraspanin constructs. These experiments revealed that CLEC-2 interacts with CD37, but not with four other tetraspanins used as controls (CD9, CD63, CD151 or CD81), under conditions using $1 \%$ digitonin (Fig. 1A,B). Interactions preserved in $1 \%$ digitonin are associated with primary (direct) interactions between a tetraspanin and its partner proteins (Serru et al., 1999). The strength of the interaction between CD37 and CLEC-2 was comparable with two well-established primary interactions between tetraspanins and their partner proteins: CD9 with CD9P1 (also known as Prostaglandin F2 receptor inhibitor) (Charrin et al., 2001) and Tspan 14 with ADAM10 (Dornier et al., 2012; Haining et al., 2012) (Fig. 1C,D). Thus, these experiments show that CLEC-2 specifically interacts with tetraspanin CD37.

\section{CD37-deficient myeloid cells show decreased CLEC-2 surface expression and increased CLEC-2-dependent IL-6 production}

We investigated CLEC-2 membrane expression on the immune cells of $C d 37^{-/}$mice. Naïve $C d 37^{-/}$myeloid cells expressed significantly lower CLEC-2 levels compared with $C d 37^{+/+}$(wildtype, WT) splenocytes (Fig. 2A). It was reported that CLEC-2 expression was increased on myeloid cells upon in vivo lipopolysaccharide (LPS) stimulation (Lowe et al., 2015; MourãoSá et al., 2011). Therefore, we analysed CLEC-2 expression on different immune cell subsets from the spleens of WT and $C d 37^{-/-}$ mice that were stimulated with LPS in vivo. CLEC-2 membrane expression was substantially increased by LPS, but this increase was significantly lower on LPS-stimulated $C d 37^{-/-}$myeloid cells (DCs, macrophages, granulocytes), compared with WT myeloid cells (Fig. 2B,C). This was in contrast to LPS-stimulated WT and $C d 37^{-1-}$ lymphoid cells [B cells, T cells and natural killer (NK) cells] that expressed comparable CLEC-2 levels (Fig. 2C). As a control, we investigated CLEC-2 expression on mouse platelets, which do not express CD37 (Zeiler et al., 2014), and observed similar CLEC-2 expression on platelets of WT and $C d 37^{-/-}$mice (Fig. S1).

Next, we investigated whether decreased CLEC-2 expression on $C d 37^{-1-}$ immune cells had functional consequences by analysing cytokine production. $C d 37^{-/-}$splenocytes produced significantly more interleukin-6 (IL-6) compared with WT splenocytes upon stimulation with the CLEC-2 ligand rhodocytin (Fig. 2D). This was not due to a general defect of the $C d 37^{-1-}$ immune cells because stimulating these cells with phorbol myristate acetate (PMA)/ ionomycin resulted in equivalent IL-6 production compared with WT cells (Fig. 2D). Thus, the presence of CD37 is important for regulation of CLEC-2 membrane expression on myeloid cells, and CD37 inhibits CLEC-2-dependent cytokine production.

\section{CD37 controls DC migration and protrusion formation in response to podoplanin}

To investigate whether CD37 was required for the migratory capacity of CLEC-2-expressing (CLEC- $2^{+}$) DCs, we performed static adhesion and migration assays of WT and $\mathrm{Cd} 37^{-/-} \mathrm{CLEC}-2^{+}$ (LPS-stimulated) bone marrow-derived DCs (BMDCs) seeded on LECs (Fig. 3A). Adhesion of both WT and $C d 37^{-1-}$ CLEC- $2^{+}$DCs to inflamed (TNF $\alpha$-stimulated) LECs was stable for the duration of the experiment. Interestingly, the percentage of $\mathrm{Cd}_{3} 7^{-/}$DCs adhering to the inflamed LECs was reduced when compared with WT DCs (Fig. 3B). Moreover, migration velocity and mean square displacement (MSD) of DCs were significantly decreased in the absence of CD37 (Fig. 3C,D; Movies 1 and 2).

To investigate whether cell morphological changes underlying DC migration are CLEC-2 dependent, we analysed the ability of DCs to form protrusions in response to the CLEC-2 ligand podoplanin in a $3 \mathrm{D}$ collagen matrix. In response to podoplanin, the protrusion length and morphology index of WT DCs was significantly increased, compared with unstimulated cells, as previously described (Acton et al., 2012) (Fig. 4A,C,D; Fig. S2). DCs lacking CD37 were capable of forming short actin protrusions (Fig. 4A,B; Fig. S2). However, $C d 37^{-/}$DCs, despite expressing similar levels of CLEC-2 (Fig. S3), were unable to increase protrusion length and morphology index in response to podoplanin, instead phenocopying the defect seen in DCs lacking CLEC-2 [CD11 c ${ }^{\Delta \mathrm{CLEC}-2}$ (Acton et al., 2012, 2014)] (Fig. 4A,C,D). Together, these results demonstrate that CD37 deficiency, similar to CLEC-2 deficiency, results in aberrant DC adhesion and migration on LNSCs, and decreased actin protrusion formation in response to the CLEC-2 ligand podoplanin.

\section{CLEC-2 recruitment to podoplanin is dependent on CD37}

To gain insight into the underlying mechanism by which CD37 controls CLEC-2 response to podoplanin, we analysed local CLEC-2 recruitment to podoplanin in the presence or absence of CD37 by microcontact printing experiments. Microcontact printing ('stamping') technology (Van Den Dries et al., 2012; Zuidscherwoude et al., 2017b) enables the imaging and analysis of CLEC-2 protein recruitment in the membrane of cells towards 
A
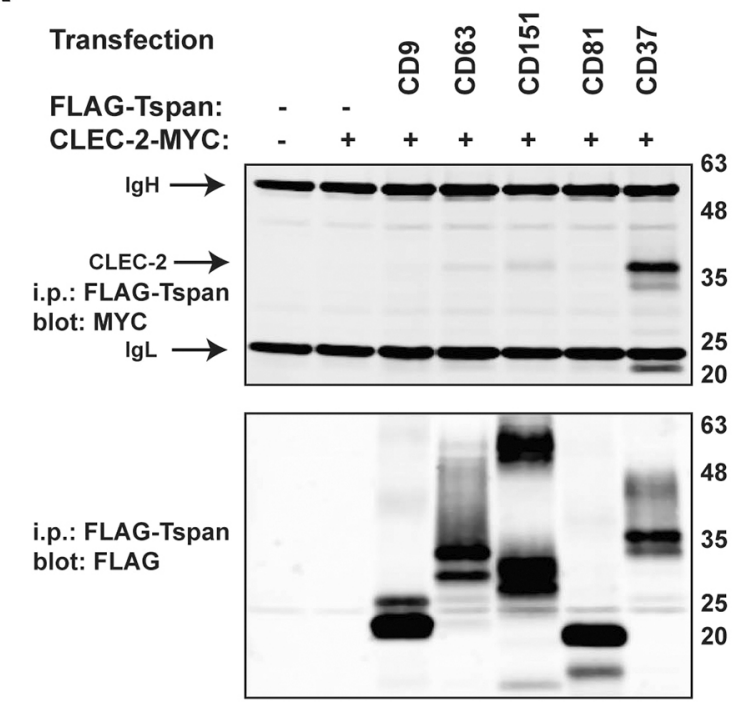

whole cell lysate blot: MYC

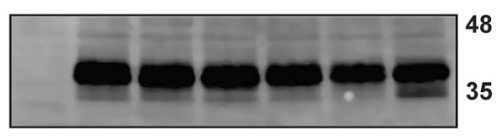

B

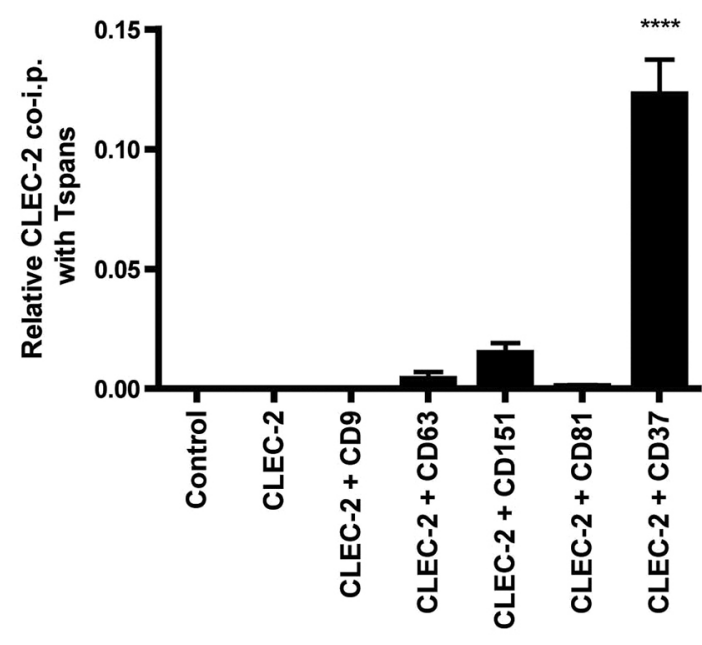

D

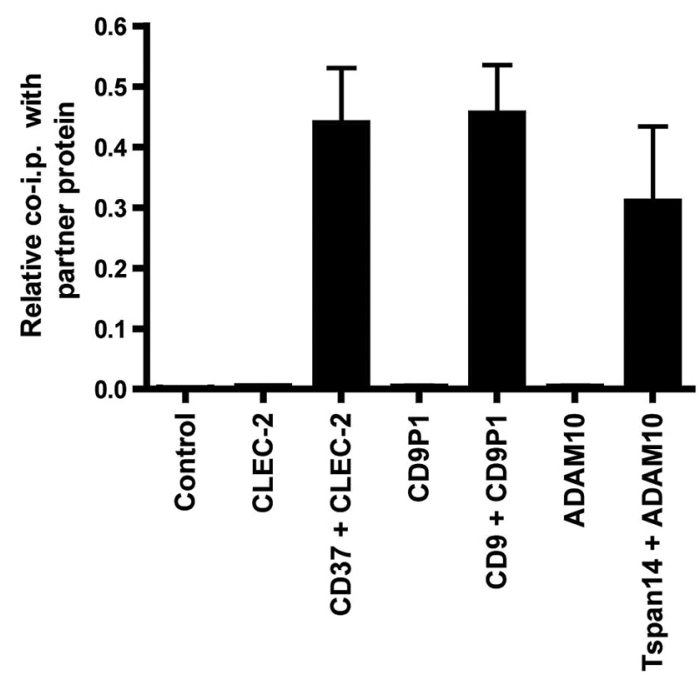

Fig. 1. CLEC-2 specifically interacts with tetraspanin CD37. (A) HEK-293T cells were co-transfected with MYC-tagged human CLEC-2 and FLAG-tagged human tetraspanins (CD9, CD63, CD151, CD81 or CD37), or mock transfected (-). Cells were lysed in 1\% digitonin and immunoprecipitated with an anti-FLAG antibody. Immunoprecipitated proteins were blotted with anti-MYC antibody (top) or anti-FLAG antibody (middle). Whole cell lysates were probed with the anti-MYC antibody (bottom). (B) Quantification of A; the amount of MYC-tagged CLEC-2 co-precipitated was normalized to the amount of tetraspanin on the beads. Data are mean+s.e.m. from three independent experiments. Data were normalized by log transformation and statistically analysed using one-way ANOVA with a Tukey's multiple comparison test, compared with the mean of every other column $\left({ }^{* * * *} P<0.0001\right)$. (C) HEK-293T cells were co-transfected with MYC-tagged human CLEC-2, CD9P1 or ADAM10 expression constructs and FLAG-tagged CD37, CD9 or Tspan14 tetraspanins, or mock transfected (-).

Cells were lysed in $1 \%$ digitonin and immunoprecipitated with an anti-FLAG antibody. Immunoprecipitated proteins were blotted with anti-MYC antibody (top) or anti-FLAG antibody (middle). Whole cell lysates were probed with the anti-MYC antibody (bottom). (D) Quantification of C; the amount of MYC-tagged partner co-precipitated was normalized to the amount of tetraspanin on the beads. Data are mean+s.e.m. from three independent experiments. 
A
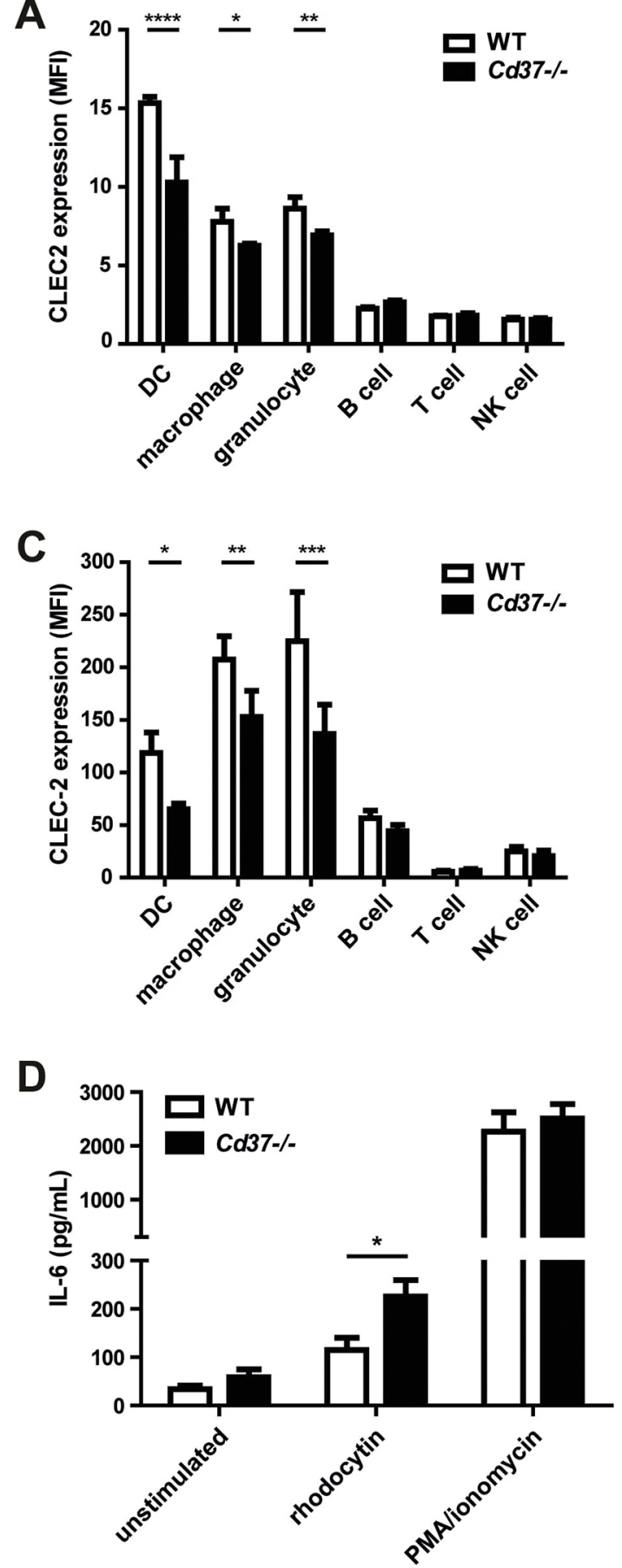

B
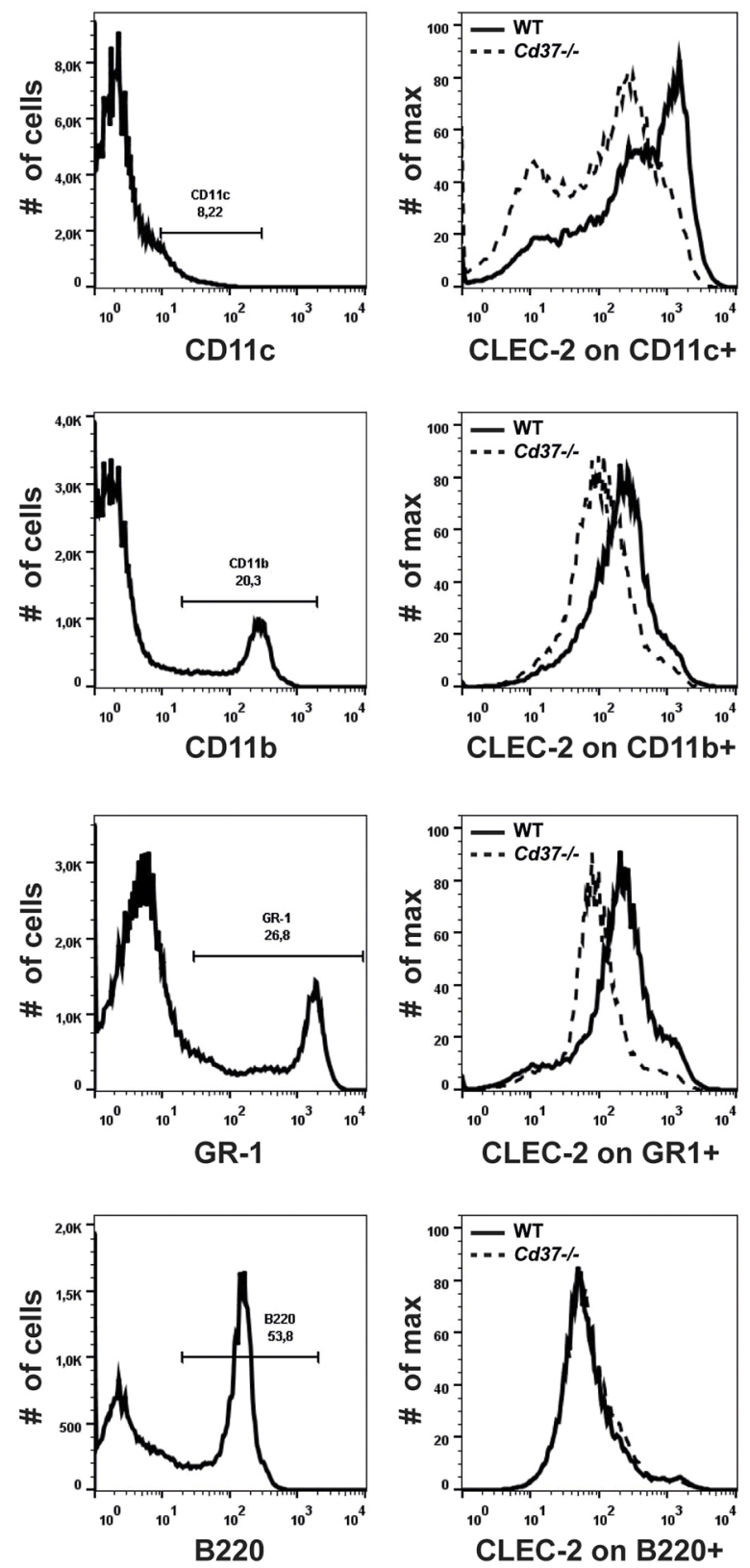

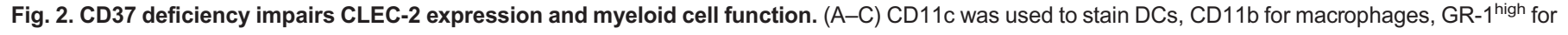
granulocytes, B220 for B cells, CD3ع for T cells and NK1.1 for NK cells. (A) Quantification of flow cytometric analysis of CLEC-2 expression of naïve WT

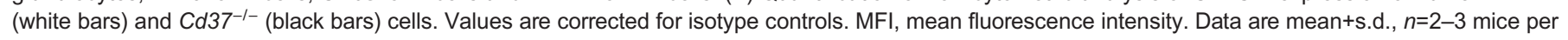
genotype. Two-way ANOVA with Sidak's multiple comparisons test, ${ }^{*} P<0.05,{ }^{* *} P<0.01,{ }^{* * *} P<0.0001$. (B) Flow cytometric analysis of CLEC-2 expression (antibody clone INU-1) on splenic immune cell subsets in WT (solid line) and Cd37-/- (dashed line) mice $24 \mathrm{~h}$ post-intraperitoneal injection with LPS.

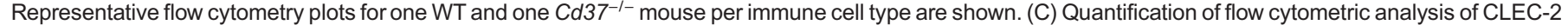
expression of in vivo LPS-stimulated WT (white bars) and $C d 37^{-1-}$ (black bars) cells. Values are corrected for isotype controls. Data are mean+s.d., $n=2-3$ mice per genotype. Two-way ANOVA with Sidak's multiple comparisons test, ${ }^{*} P<0.05,{ }^{* *} P<0.01,{ }^{* * *} P<0.001$. (D) IL-6 production (in pg/ml) by total splenocytes from naïve WT (white bars) and Cd37-/- (black bars) mice after ex vivo stimulation with medium (unstimulated, negative control), 15 $\mu$ g/ml rhodocytin (CLEC-2 agonist) or PMA/ionomycin (positive control). Data are mean+s.e.m. from three independent experiments, $n=6-8$ mice per genotype. Nonparametric Mann-Whitney test, two-tailed, ${ }^{*} P=0.0215$.

recombinant podoplanin protein that is stamped as circular spots $(5 \mu \mathrm{m})$ on glass coverslips. Myeloid cells (RAW macrophages) were transiently transfected with murine CLEC- 2 tagged to GFP
(GFP-mCLEC-2) with or without murine CD37 tagged to mCherry (mCD37-mCherry) (Fig. 5A; Fig. S4A), and incubated on coverslips with podoplanin stamps to locally engage CLEC-2 
A
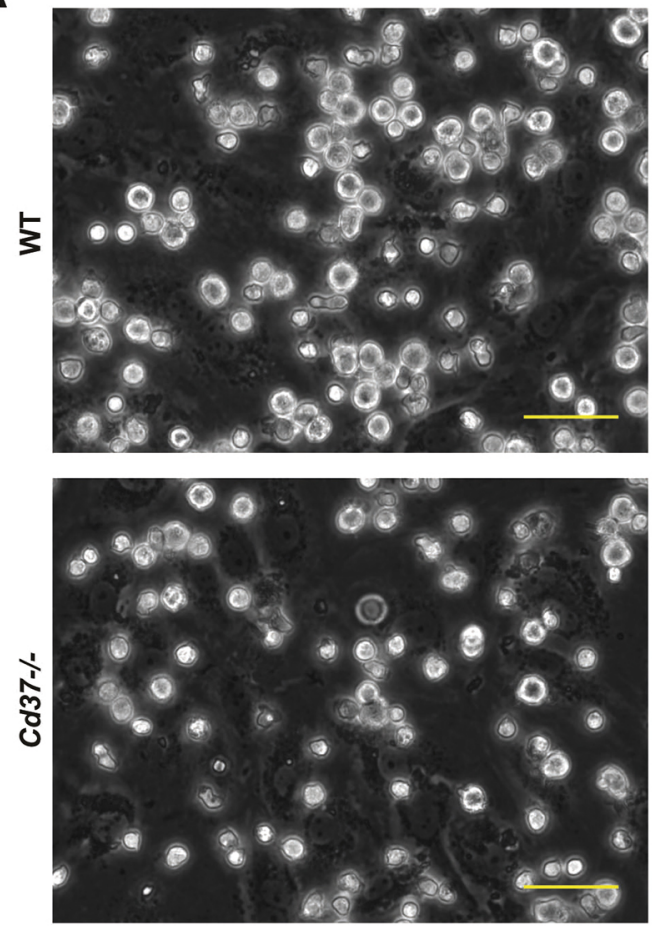

B

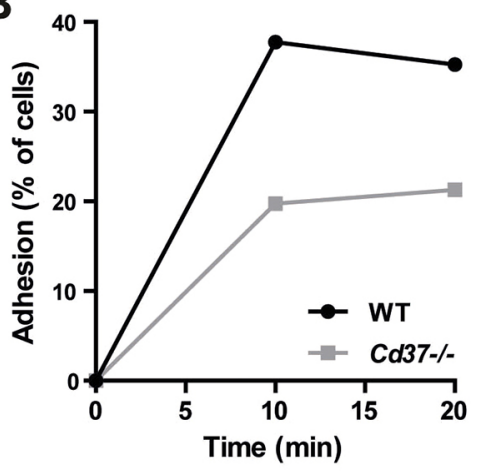

C

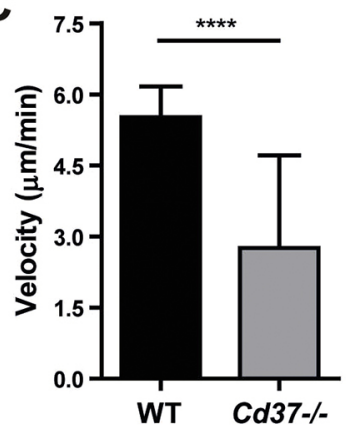

Fig. 3. CD37 controls DC adhesion, migration velocity and displacement on lymphatic endothelial cells (LECs). (A) Adherence of WT (top) and $\mathrm{Cd} 37^{-/-}$ (bottom) DCs on TNF $\alpha$-stimulated LECs. One representative image per genotype is shown Scale bars: $50 \mu \mathrm{m}$. (B) Adhesion (\% of total cells added per well) of WT (black line) and Cd $37^{-/-}$(grey line) DCs on LECs for the duration of the experiment. Data shown are from one representative experiment.

Experiments were repeated two times yielding similar results. (C) Migration velocity $(\mu \mathrm{m} / \mathrm{min})$ of WT (black bar) and Cd37-l- (grey bar) DCs over LECs. Bars represent median and interquartile range from two independent experiments, $n=107-115$ cells per genotype. Nonparametric Mann-Whitney test, twotailed, ${ }^{* * * *} P<0.0001$. (D) Left: zoom of field of view shown in A with individual cell tracks [WT (top), $\mathrm{Cd}_{37^{-1-}}$ (bottom)]. Tracking paths of each cell of one representative experiment are shown in Movies 1 and 2. Scale bars: $25 \mu \mathrm{m}$. Right: mean square displacement (MSD, in $\mu \mathrm{m}^{2}$ ) of WT (black line) and $\mathrm{Cd} 37^{-/-}$(grey line) DCs on LEC. Data are mean \pm s.e.m. from two independent experiments. Two-way ANOVA with Sidak's multiple comparisons, ${ }^{* * * *} P<0.0001$ at $\mathrm{t}=5 \mathrm{~min}$
D
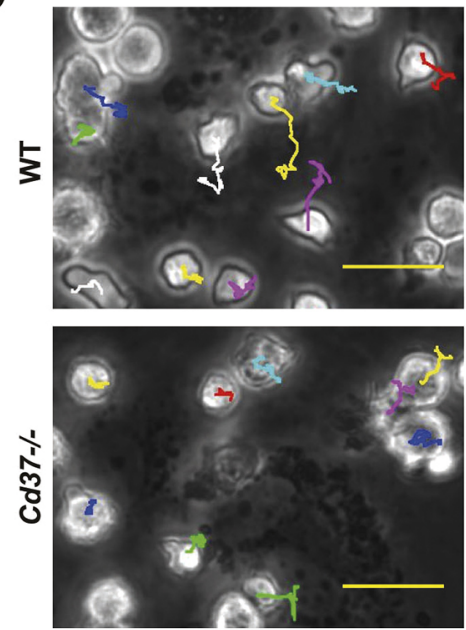

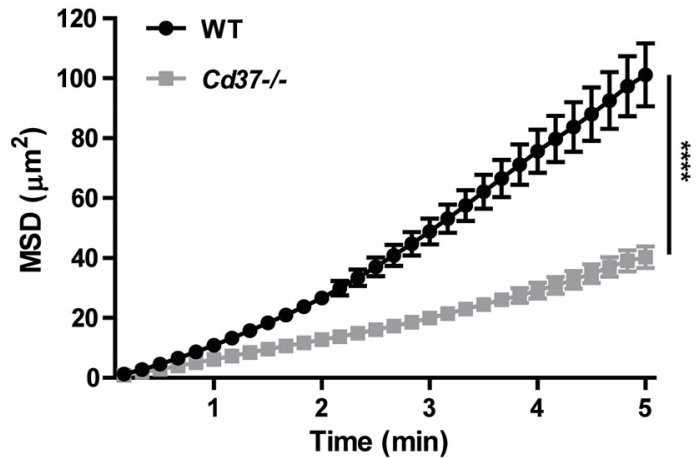

molecules at sites of podoplanin. We first determined CLEC-2 membrane expression in the transfected cells to rule out differences in transfection efficiency. CLEC-2 membrane expression was comparable between cells transfected with or without mCD37mCherry (Fig. 5B). Cells expressing both GFP-mCLEC-2 and mCD37-mCherry showed $a>2$-fold higher percentage of cells with local (on-stamp) CLEC-2 enrichment at sites of podoplanin, compared with cells only expressing GFP-mCLEC-2 (Fig. 5C,D; Fig. S4B). These data indicate that CD37 significantly facilitates CLEC-2 recruitment to podoplanin.

\section{CD37 is not required for interaction between two different CLEC-2 molecules}

Signalling downstream of CLEC-2 requires dimerization of CLEC-2 upon ligand (podoplanin) binding (Fuller et al., 2007; Hughes et al., 2010; Suzuki-Inoue et al., 2006). We hypothesized that CD37 drives CLEC-2 recruitment to podoplanin by facilitating the interaction between different CLEC-2 molecules. To study this, we transfected two differently tagged human CLEC-2 constructs (MYC-hCLEC-2 and FLAG-hCLEC-2) into HEK-293T cells with or without co-transfecting human $\mathrm{CD} 37$, and subsequently performed coimmunoprecipitation experiments with an anti-FLAG antibody under conditions using $1 \%$ digitonin. There was no difference in the amount of MYC-hCLEC-2 co-immunoprecipitated with FLAGhCLEC-2 in the absence or presence of CD37 (Fig. 6A,B). Flow cytometry confirmed the successful transfection of CD37 (Fig. 6C). This result suggests that $\mathrm{CD} 37$ does not control the interaction between two different CLEC-2 molecules.

\section{CD37 is important for CLEC-2-induced loss of actomyosin} contractility in FRCs upon interaction with podoplanin

We next investigated whether CD37 was involved in the interaction between CLEC-2 and podoplanin. Therefore, we performed co-culture experiments with CLEC-2 ${ }^{+}$DCs present in a $3 \mathrm{D}$ 
A
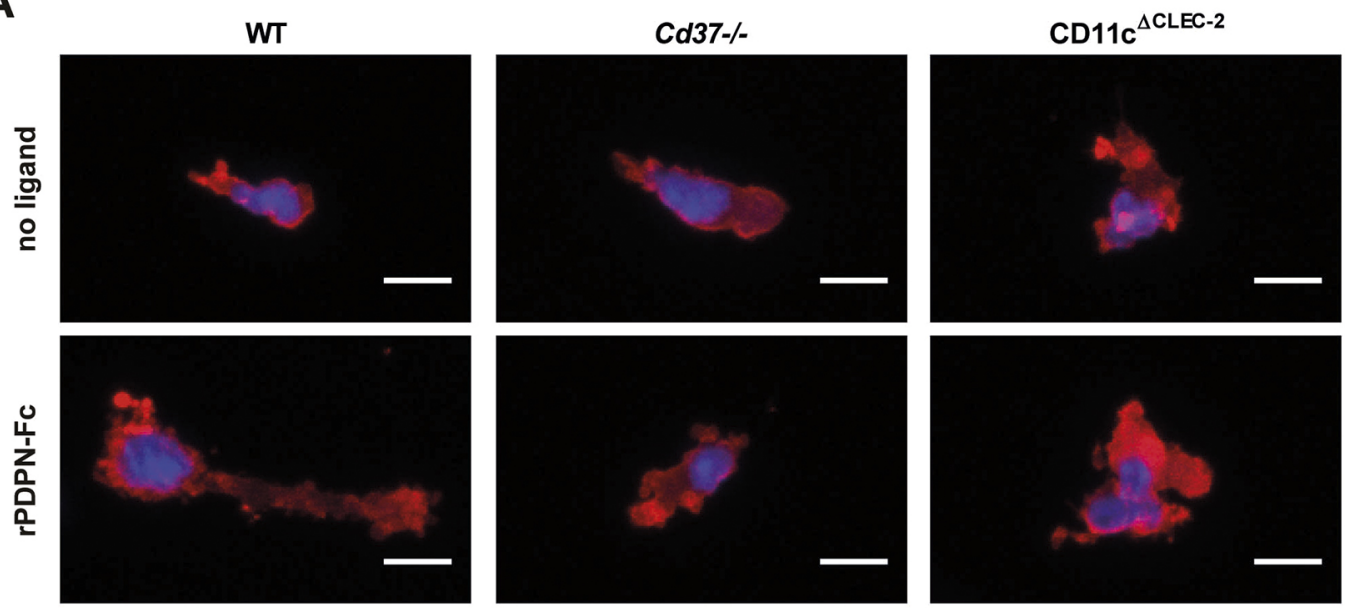

B

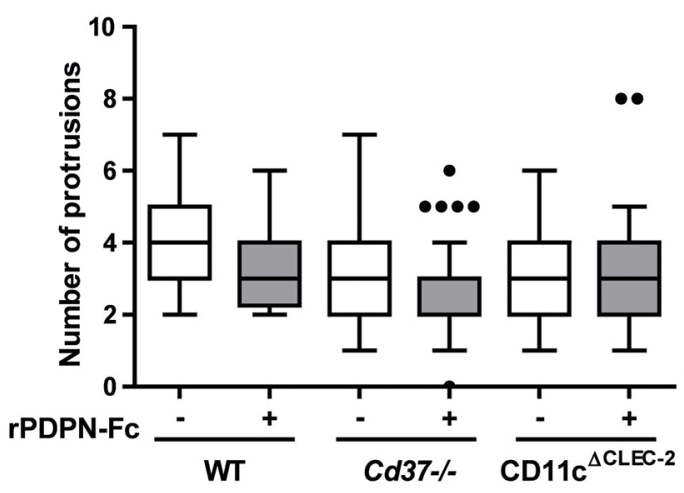

C

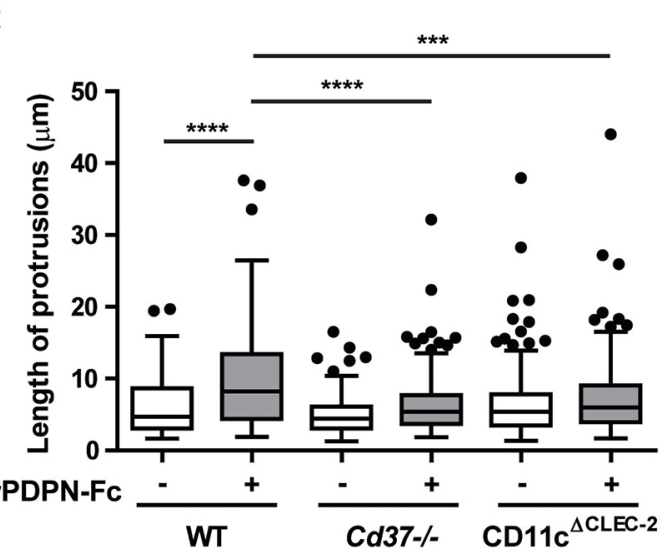

D

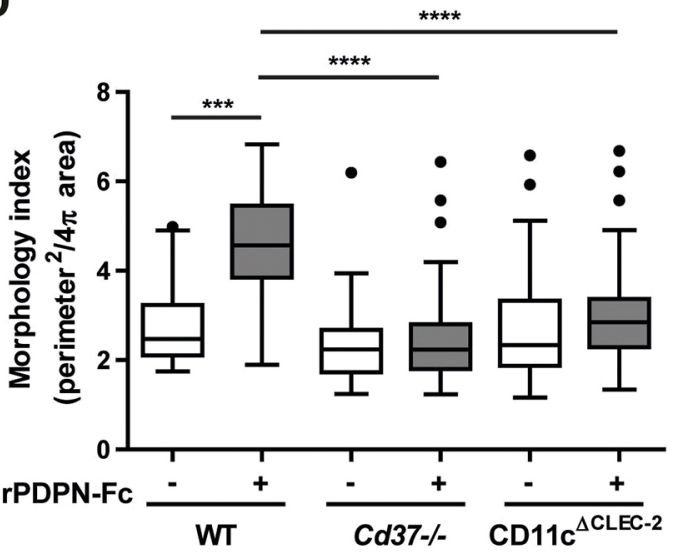

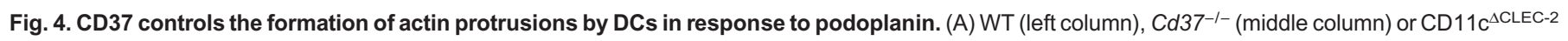

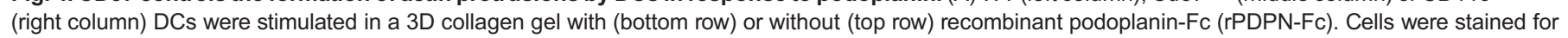
F-actin (red) and nuclei (blue) and imaged with a Leica SP5 confocal fluorescence microscope. One representative cell is shown for each condition (overview with more cells is provided in Fig. S2). Scale bars: $10 \mu \mathrm{m}$. (B-D) Number (B) and length (C) of actin protrusions, and morphology index (D) of WT (left), Cd37 ${ }^{-/-}$ (middle) or CD11 $\mathrm{c}^{\triangle \mathrm{CLEC}-2}$ (right) BMDCs upon rPDPN-Fc stimulation (grey boxes) compared with no ligand (white boxes). Data are shown as Tukey box-andwhiskers plots from three independent experiments, $n=41-67$ cells. In Tukey box-and-whiskers plots, outliers are represented as black dots; i.e. data points outside the 25 th and 75 th percentile, minus or plus the 1.5 interquartile range, respectively. Two-way ANOVA with Tukey's multiple comparisons, ${ }^{\star * \star} P<0.001$, $* * * * P<0.0001$.

collagen matrix on top of a monolayer of podoplanin-expressing FRCs. $C d 37^{-/-}$DCs had a significantly lower morphology index compared with WT DCs upon interaction with FRCs, similar to findings of DCs cultured in a 3D collagen matrix with podoplanin
(Fig. 4), phenocopying the effect observed with CD11 $\mathrm{c}^{\Delta \mathrm{CLEC}-2} \mathrm{DCs}$ (Fig. 7A,B).

Besides being a receptor for podoplanin, CLEC-2 can also induce reciprocal signalling upon interaction with podoplanin, resulting in 
A

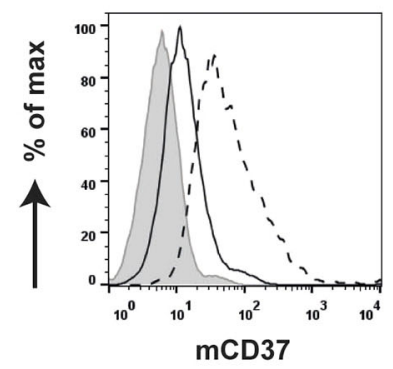

C


B
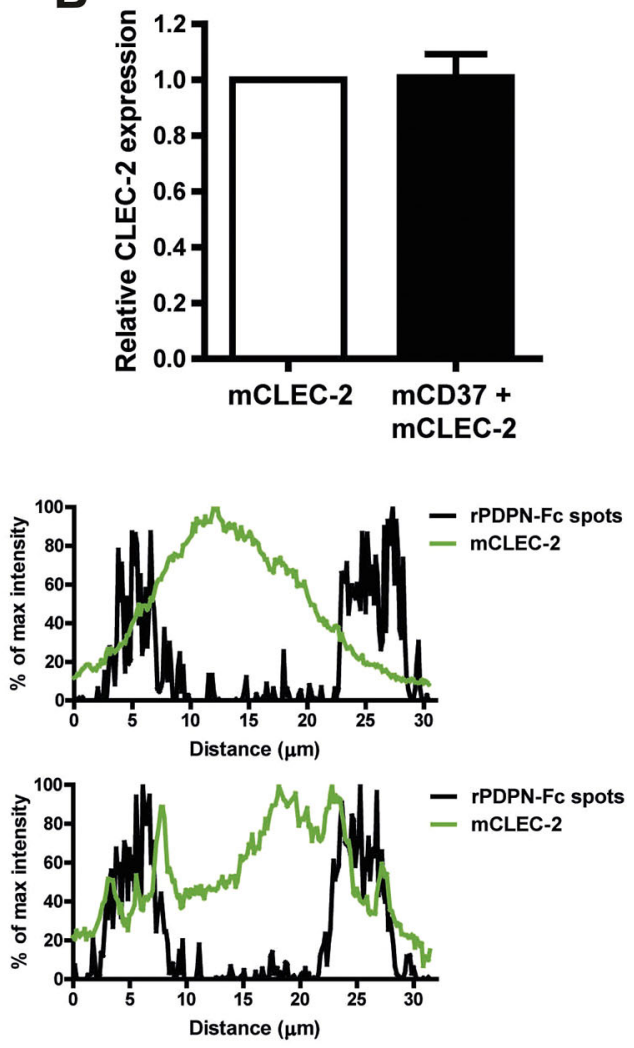

D

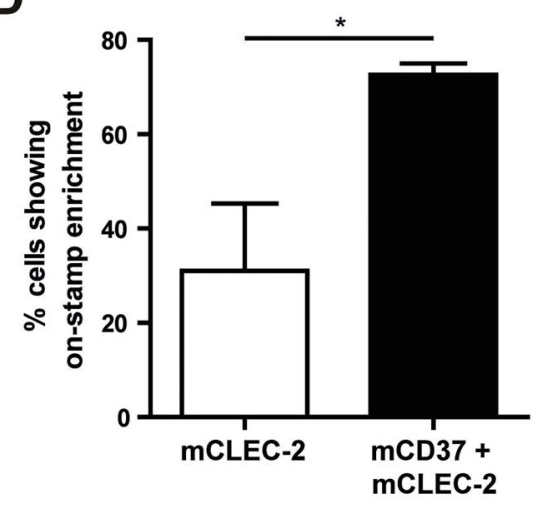

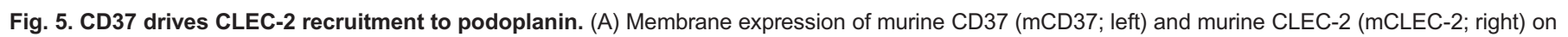

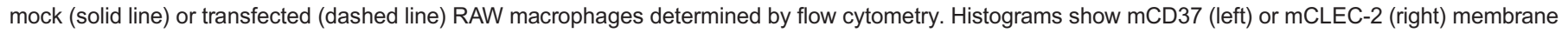

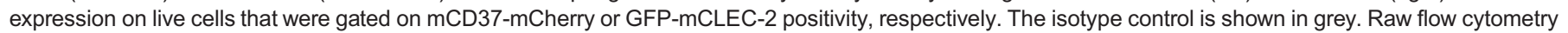

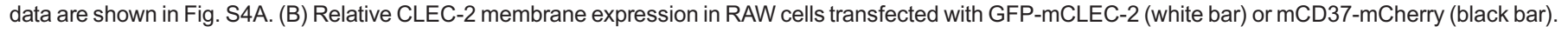

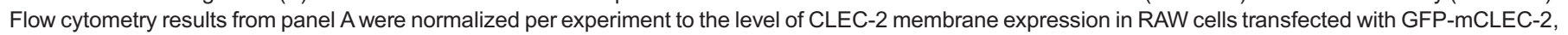

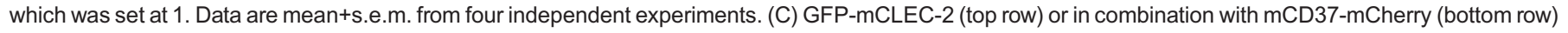
transfected RAW macrophages were incubated on recombinant podoplanin-Fc (rPDPN-Fc) spots and analysed after 12 min using an epi-fluorescence

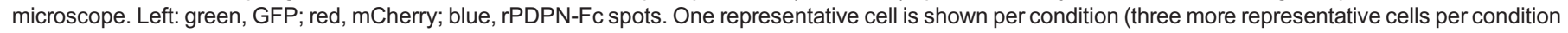
are shown in Fig. S4B). Scale bars: $10 \mu \mathrm{m}$. Right: graphs represent the intensity profile of GFP-mCLEC-2 (green line) or rPDPN-Fc spot (black line) across the respective yellow lines shown on the left. (D) Percentage of GFP-mCLEC-2 (white bar) or in combination with mCD37-mCherry (black bar) transfected RAW macrophages showing enrichment of GFP-mCLEC-2 on rPDPN-Fc spots. Data are mean+s.e.m. from three independent experiments, $n=54-56$ cells. Paired Student's $t$-test, one-tailed, ${ }^{*} P=0.0372$.

inhibition of actomyosin contractility in FRCs (Acton et al., 2014). We next studied the effect of $C d 37^{-/-}$DCs on FRC contractility by measuring filamentous actin (F-actin), which forms stress fibres, using phalloidin immunofluorescence staining. FRCs interacting with WT DCs showed a dramatic decrease in actin stress fibres compared with FRCs not interacting with DCs (Fig. 7A,C). In contrast, FRCs interacting with $C d 37^{-/}$DCs showed limited reduction in actin stress fibres, similar to the effect seen in FRCs interacting with CD11 $\mathrm{c}^{\triangle \mathrm{CLEC}-2}$ DCs (Fig. 7A,C). Taken together, our data demonstrate that CD37 facilitates CLEC-2-dependent DC morphology and migration, and controls reciprocal signalling upon CLEC-2 interaction with podoplanin on FRCs (Fig. 8). 

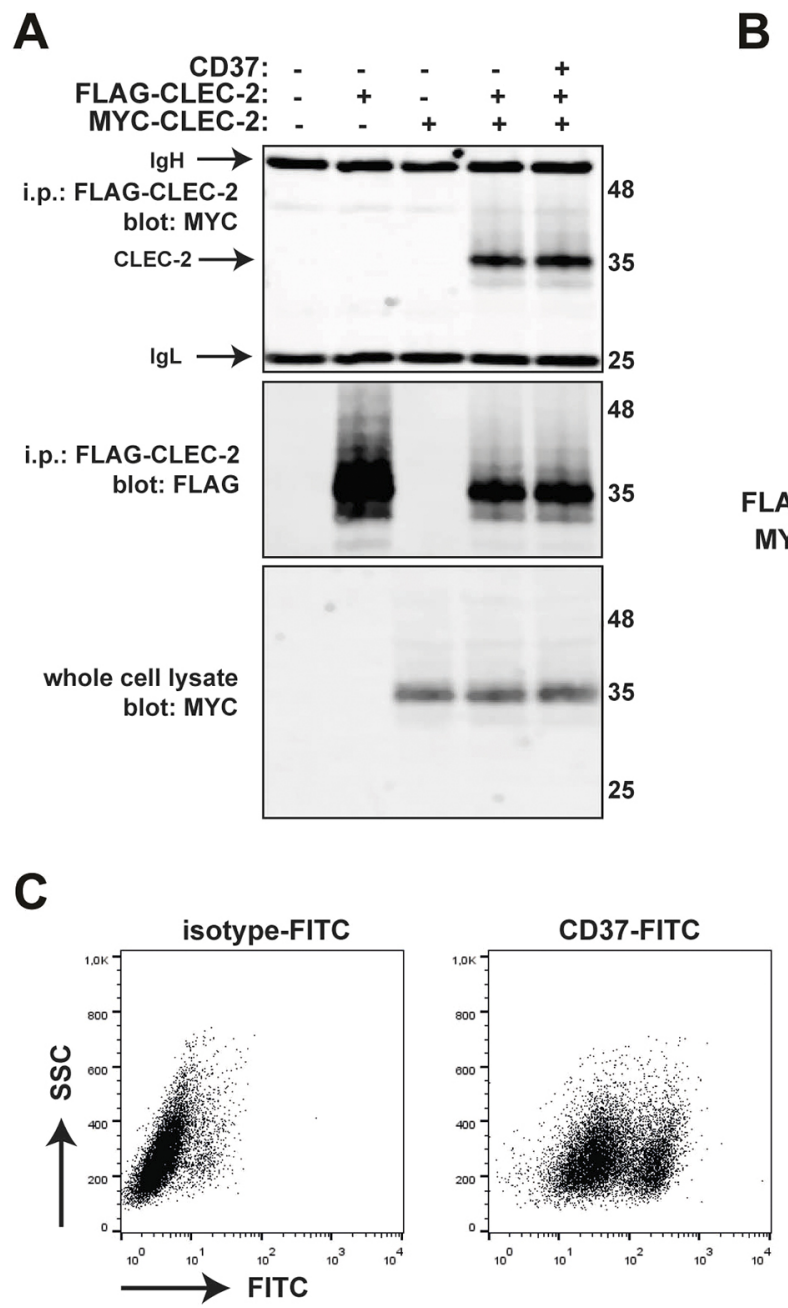

B

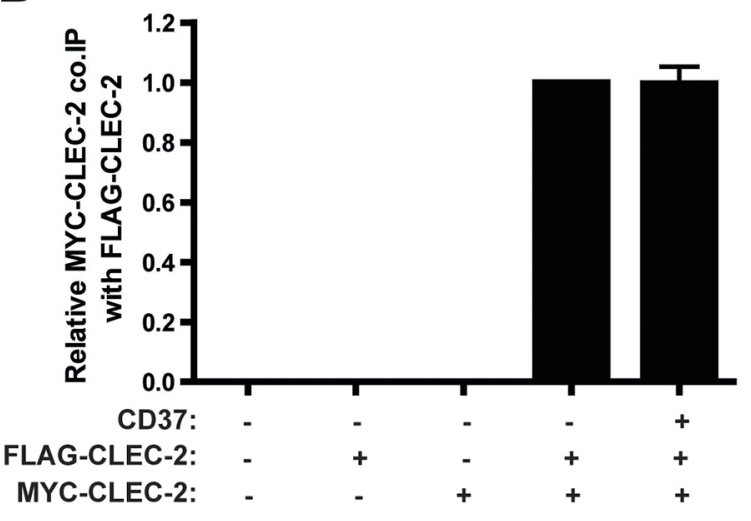

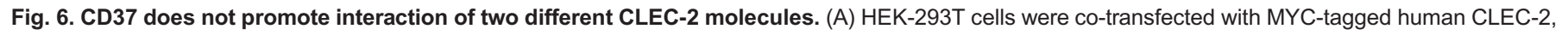
FLAG-tagged human CLEC-2, human CD37 or mock transfected (-). Cells were lysed in 1\% digitonin and immunoprecipitated with an anti-FLAG antibody. Immunoprecipitated proteins were blotted with anti-MYC (top panel) or anti-FLAG antibody (middle panel). Whole cell lysates were probed with anti-MYC

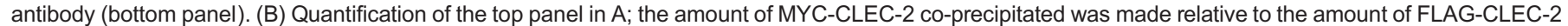
on the beads. Data are mean+s.e.m. from three independent experiments. (C) Transfected HEK-293T cells from A and B were stained with FITC-conjugated anti-CD37 antibody, or isotype control, and analysed by flow cytometry. Data shown are representative dotplots of side scatter (SSC) versus FITC fluorescence for three independent experiments.

\section{DISCUSSION}

It is well established that CLEC-2 interaction with podoplanin is essential for DC migration and initiation of the cellular immune response (Acton et al., 2012, 2014; Astarita et al., 2015); however, the underlying molecular mechanisms remain elusive. We identified a novel molecular interaction between CLEC-2 and CD37, which was specific for CD37, as other tetraspanins (CD9, CD63, CD81 and CD151) did not interact with CLEC-2. We discovered that DCs lacking CD37 have decreased CLEC-2 expression at the cell surface, and impaired adhesion, migration velocity and displacement on podoplanin-expressing lymph node stromal cells. Moreover, podoplanin-induced formation of actin protrusions by DCs and recruitment of CLEC-2 on RAW macrophages to podoplanin was impaired in the absence of CD37. Our data from DC-FRC co-culture experiments indicate that CD37 stabilizes CLEC-2 protein clusters that bind podoplanin, which not only induces DC adhesion, but also facilitates inhibition of actomyosin contractility in FRCs (Fig. 8).

For efficient ligand binding and activation of downstream signalling, CLRs have been postulated to depend on spatiotemporal localization into specific microdomains on the plasma membrane (Cambi et al., 2004; Figdor and van Spriel, 2009; Meyer-Wentrup et al., 2007). CLEC-2 has been found to be present in clusters on blood platelets (Hughes et al., 2010; Pollitt et al., 2014) and CLEC-2 clusters were reported to be localized in lipid rafts (Manne et al., 2015; Pollitt et al., 2010) by using detergentresistant membrane extraction. However, this technique also enriches for tetraspanin microdomains (Blank et al., 2007; Claas et al., 2001; Tarrant et al., 2003). We here identified a specific molecular interaction between CLEC-2 and tetraspanin CD37, indicating that CD37 microdomains form the scaffold for CLEC-2 clusters on the plasma membrane of DCs, although our data indicate that CD37 was not required for the interaction between two CLEC-2 molecules. The finding that CLEC-2 did not interact with other tetraspanins might be explained by recent super-resolution studies of the tetraspanin web, in which individual tetraspanins were found in separate nanoclusters (100-120 nm) at the cell surface of B cells and DCs (Zuidscherwoude et al., 2015). Our data are in line with the demonstration that expression and stabilization of the CLEC-2 homologous family member Dectin-1 at the plasma membrane of 
A
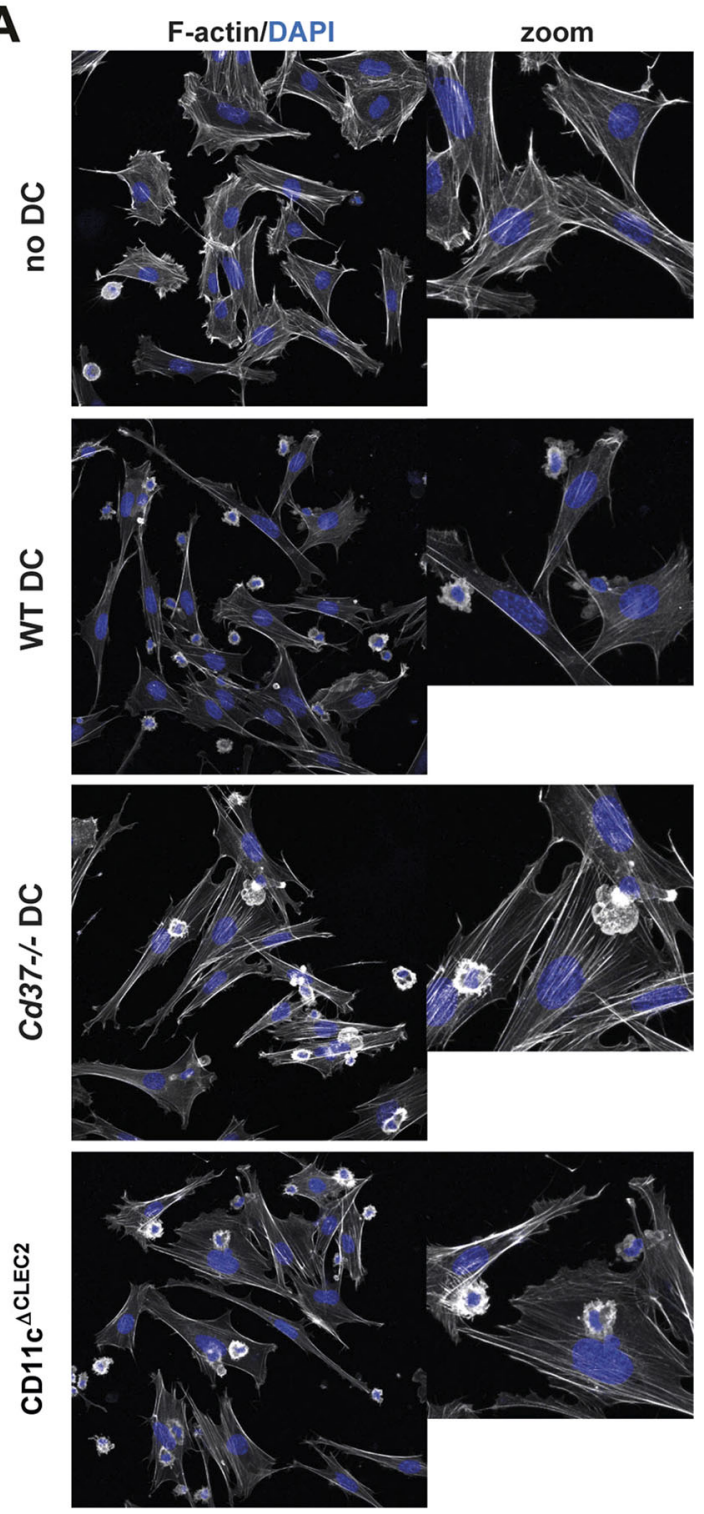

B

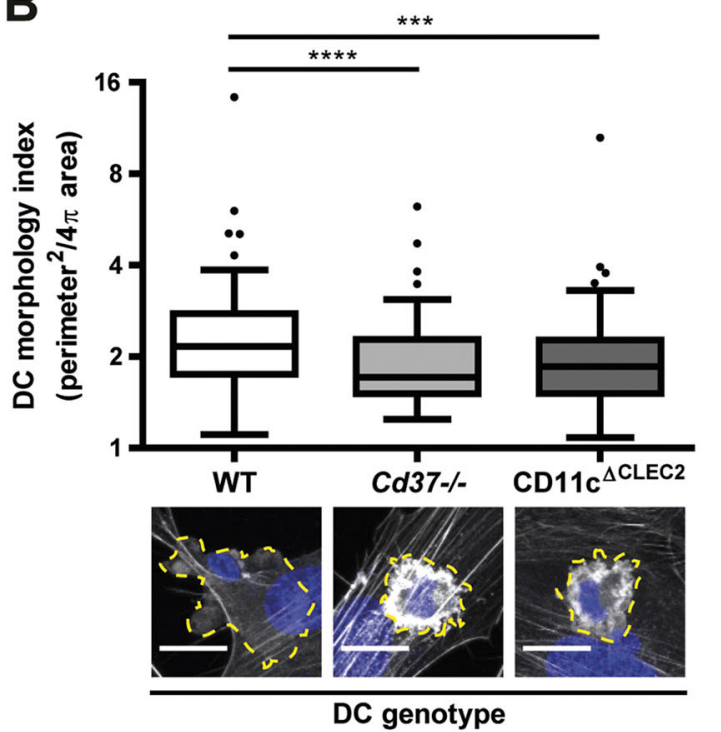

F-actin/podoplanin/DAPI
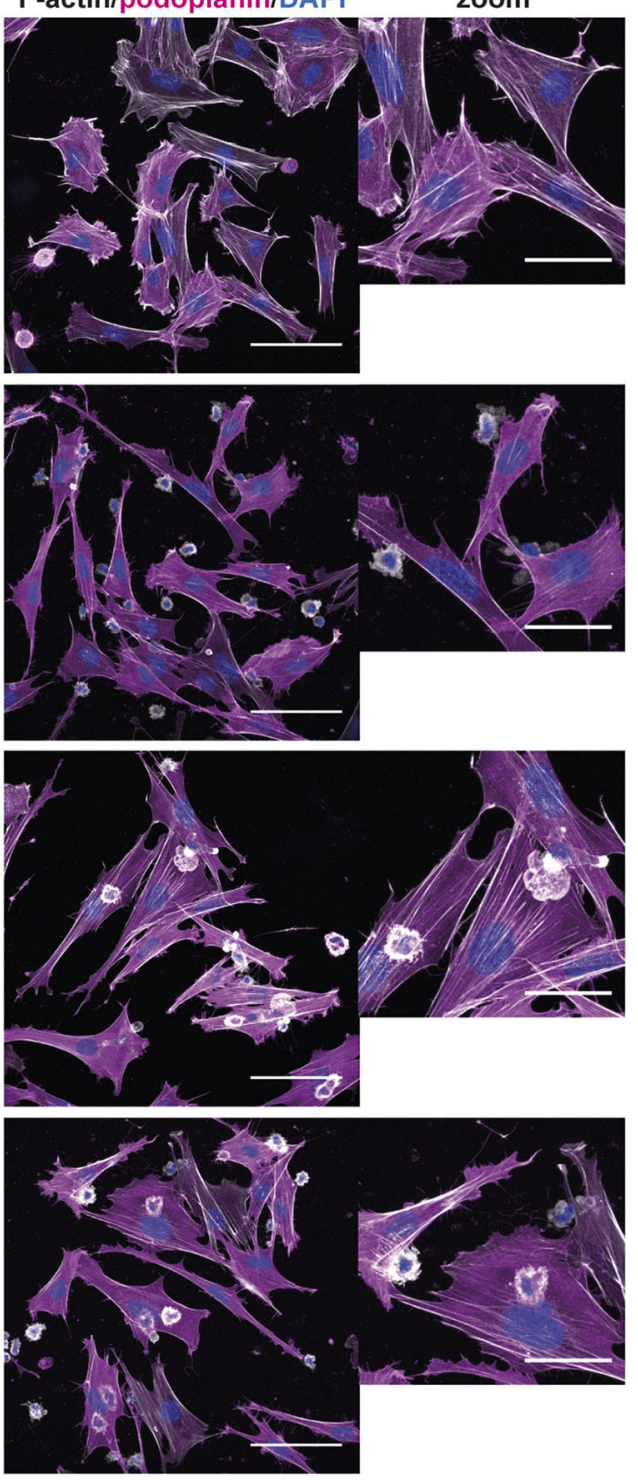

C

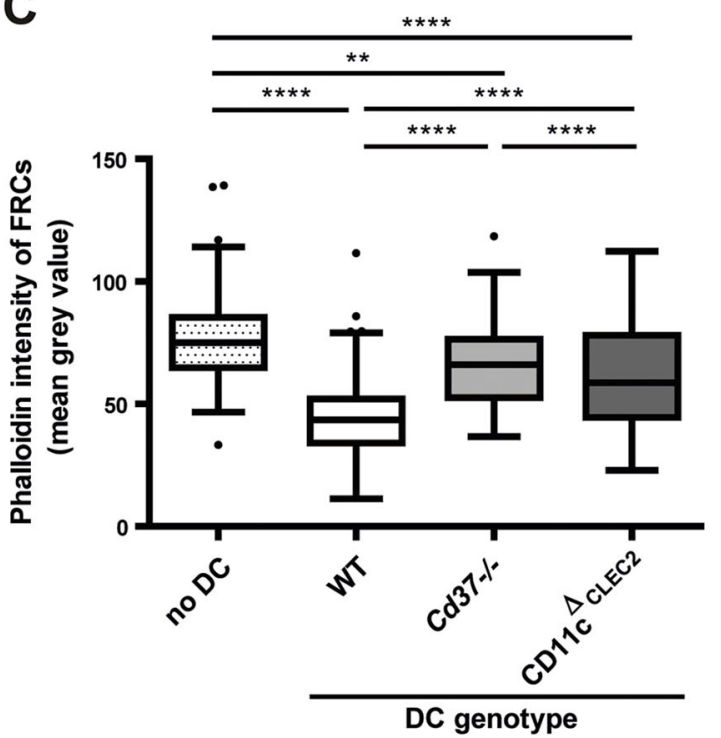

Fig. 7. See next page for legend. 
Fig. 7. CD37 expression is required for CLEC-2-induced loss of actomyosin contractility upon interaction with podoplanin on FRCs. (A) WT (second row), Cd37-l- (third row) or CD11c $\triangle$ CLEC-2 (bottom row) LPS-stimulated BMDCs were cultured in a 3D collagen matrix on top of a FRC monolayer for $24 \mathrm{~h}$. A 3D collagen matrix without DCs on top of the FRCs was used as a control (no DC, top row). Cells were stained for F-actin (white/grey), podoplanin (magenta) and nuclei (blue), and imaged with a Leica SP5 confocal fluorescence microscope. Scale bars: $100 \mu \mathrm{m}$ (overview) or $50 \mu \mathrm{m}$ (zoom). (B) Morphology index of WT (white), Cd37-l- (light grey) or CD11c $\triangle$ CLEC-2 (dark grey) BMDCs interacting with FRCs. The $y$-axis is presented as a Log2 scale. One representative BMDC for each genotype is shown below the box-and-whisker plots. The yellow dashed line indicates the cell outline used to calculate the morphology index. Scale bars: $20 \mu \mathrm{m}$. (C) Phalloidin intensity (mean grey value) of FRCs interacting with WT (white), $C d 37^{-1-}$ (light grey) or CD11c $\Delta$ CLEC-2 (dark grey) BMDCs. Phalloidin intensity of FRCs without DCs in a separate culture condition was taken as the control (dotted). Data in B and C are shown as Tukey box-and-whiskers plots from three independent experiments, $n=3$ mice per genotype, $n=71-134$ cells. In Tukey box-and-whiskers plots, outliers are represented as black dots (each dot represents an individual cell); i.e. data points outside the 25th and 75th percentile, minus or plus the 1.5 interquartile range, respectively. Kruskal-Wallis one-way ANOVA test with Dunn's multiple comparisons ${ }^{* *} P<0.01,{ }^{* *} P<0.001,{ }^{* * *} P<0.0001$

macrophages was dependent on CD37 (Meyer-Wentrup et al., 2007). It has previously been shown that CLEC-2 is internalized upon ligand binding (Acton et al., 2012). Our finding that CLEC-2 surface expression is decreased on $C d 37^{-1-}$ splenocytes compared with WT splenocytes might indicate that CLEC-2 turnover upon binding to podoplanin on LNSCs during in vivo migration is increased in the absence of CD37. CLEC-2 ${ }^{+}$WT and $C d 37^{-1-}$ BMDCs did not encounter any CLEC-2 ligand during culture and differentiation, which might explain why CLEC-2 expression was not decreased in $C d 37^{-/-}$compared with WT BMDCs

Our results show that CLEC-2 $2^{+} C d 37^{-/}$DCs have an impaired capacity to adhere to podoplanin-expressing LECs, and demonstrate lower migration velocity and displacement. This is in accordance with studies showing impaired migration of $C d 37^{-/}$DCs in vivo (Gartlan et al., 2013; Jones et al., 2016). These studies showed decreased spreading and adhesion of BMDCs cultured on fibronectin, a ligand for $\alpha 4 \beta 1$ integrin. As CD37 interacts with $\alpha 4 \beta 1$ integrin (van Spriel et al., 2012), it was postulated that impaired DC adhesion and migration in the absence of CD37 could be explained by defective $\alpha 4 \beta 1$ integrin signalling (Gartlan et al., 2013; Jones et al., 2016). Here, we demonstrate a specific role for CD37 in controlling CLEC-2 function in the migration of DCs. $C d 37^{-/}$DCs show impaired actin protrusion formation upon podoplanin stimulation, which is highly similar to the phenotype of CD11 $\mathrm{c}^{\triangle \mathrm{CLEC}-2}$ DCs. Rearrangements of the actin cytoskeleton and subsequent cell movement are controlled by Rho GTPases, including RhoA and Rac1. RhoA increases actomyosin contractility via its interaction with Rho kinases (Parri and Chiarugi, 2010), whereas Rac1 supports actin polymerization, spreading of lamellipodia and formation of membrane ruffles (Nobes and Hall, 1995; Olson and Sahai, 2009). Because the activity of the Rho GTPases has been shown to change upon CLEC-2 activation by podoplanin or rhodocytin (i.e. downregulation of RhoA and upregulation of Rac1) (Acton et al., 2012), we postulate that the underlying molecular mechanism of the defective cell migration of $C d 37^{-/}$DCs is due to deregulation of intracellular Rho GTPases as a consequence of impaired recruitment of CLEC-2 to its ligand
A

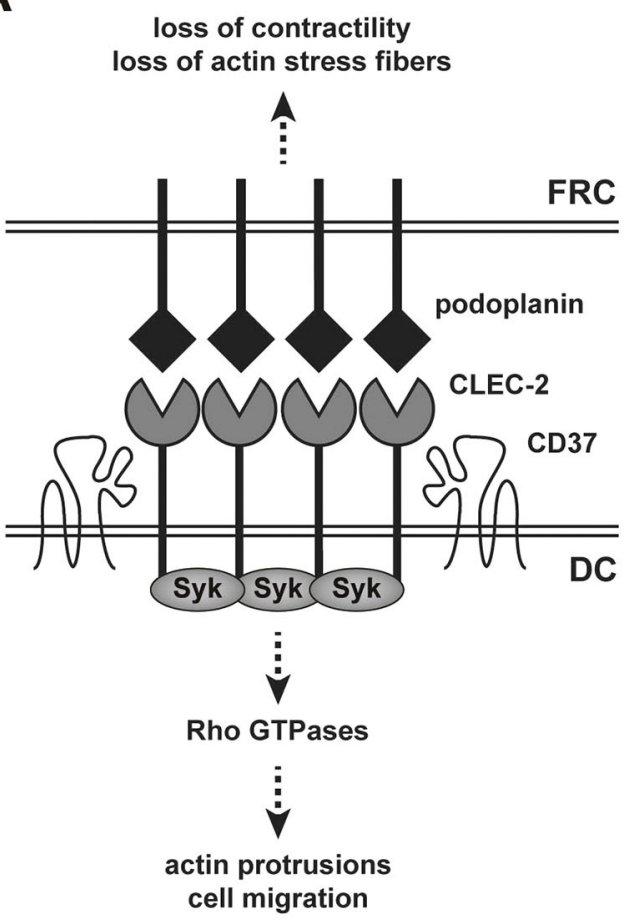

B contractile phenotype
presence of actin stress fibers

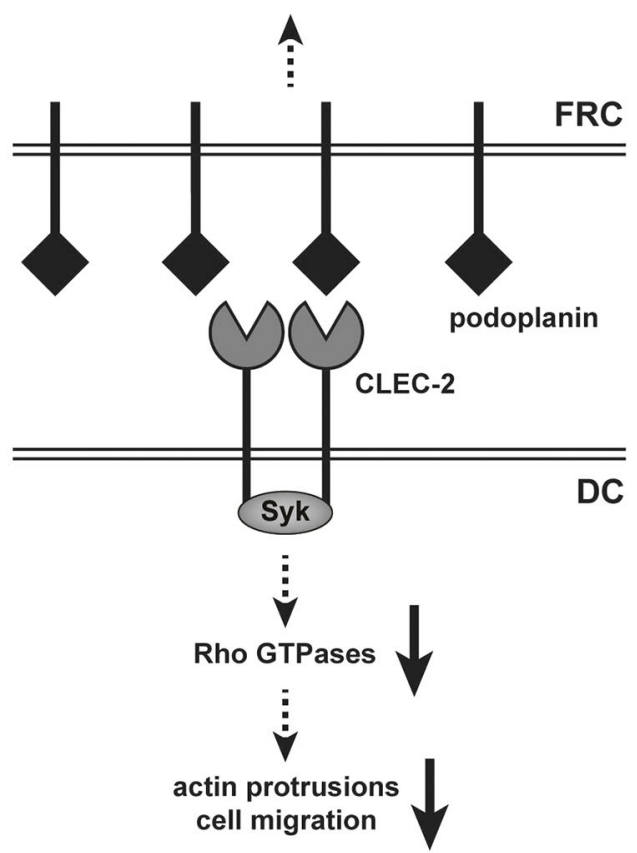

Fig. 8. Model illustrating that tetraspanin CD37 controls the function of CLEC-2 as a receptor in dendritic cell migration, and facilitates CLEC-2-induced loss of actomyosin contractility by podoplanin binding on FRCs. (A) CD37 drives the recruitment of CLEC-2 proteins in the plasma membrane upon podoplanin. As such, CD37 might regulate activation of Syk and, most likely via changes in Rho GTPase activity, this results in the formation of actin protrusions and DC migration. In addition, our data indicate that the interaction between CLEC-2 and podoplanin on FRCs is stabilized by CD37 and, as such, facilitates inhibition of FRC actomyosin contractility as measured by loss of actin stress fibres. (B) In the absence of CD37, recruitment of CLEC-2 upon podoplanin binding is impaired, resulting in decreased DC migration. Moreover, FRCs retain a contractile phenotype as showed by the presence of actin stress fibres. 
podoplanin. This is supported by a recent study demonstrating impaired activation of Racl in toxin-activated adherent $\mathrm{Cd} 37^{-/-}$ BMDCs (Jones et al., 2016). Altogether, these data support a model in which $\mathrm{CD} 37$ is important for CLEC-2 recruitment in the plasma membrane of myeloid cells upon podoplanin binding, which results in Syk activation and changes in Rho GTPase activity (e.g. increased Rac1 and decreased RhoA activation) leading to cell migration (Fig. 8).

Besides activation of Rho GTPases, CLRs can initiate intracellular Syk-dependent signalling cascades that induce cytokine production (Mócsai et al., 2010). We found that $C d 37^{-/}$ myeloid cells produce higher IL-6 levels compared with WT cells upon stimulation with the CLEC-2 ligand rhodocytin, which is in line with previous reports showing production of pro-inflammatory cytokines (i.e. IL-6 and TNF $\alpha$ ) by neutrophils and RAW macrophages upon stimulation with rhodocytin (Chang et al., 2010; Kerrigan et al., 2009). Increased IL-6 expression upon CLR stimulation has also been shown in $C d 37^{-/-}$macrophages upon stimulation with the Dectin-1 ligand curdlan (Meyer-Wentrup et al., 2007). Additionally, IL-10 production by RAW macrophages and BMDCs co-stimulated with LPS and anti-CLEC-2 Fab fragments could be reversed by Syk inhibition (Mourão-Sá et al., 2011). Our results suggest that CD37 directly controls Syk signalling downstream of hemITAM CLRs and, as such, inhibits cytokine production, probably by stimulating phosphatase activity (Carmo and Wright, 1995; Chattopadhyay et al., 2003; Wright et al., 2004). Syk activation has been reported to be negatively regulated by $\mathrm{SH} 2$ domain-containing protein tyrosine phosphatase 1 (SHP1; also known as PTPN6) (Mócsai et al., 2010; Zhang et al., 2000). SHP1 and CD37 have been shown to associate via the N-terminal immunoreceptor tyrosine-based inhibition motif (ITIM)-like domain of CD37 in chronic lymphocytic leukaemia cells, which induced tumour cell death via negative regulation of AKT (also known as AKT1)-mediated pro-apoptotic signalling (Lapalombella et al., 2012). The binding of cytoplasmic signalling proteins, like protein kinase C (PKC) (Zhang et al., 2001; Zuidscherwoude et al., 2017b), Rac1 (Tejera et al., 2013) and suppressor of cytokine signaling 3 (SOCS3) (de Winde et al., 2016), has been reported for different tetraspanins (also reviewed in van Deventer et al., 2017).

In conclusion, our results demonstrate that CD37 is required for ligand-induced CLEC-2 responses via a direct molecular interaction, leading to immune cell activation and DC migration. Furthermore, CD37 controls the interaction of CLEC-2 with podoplanin, resulting in inhibition of FRC actomyosin contractility. This study supports a general mechanism of tetraspanin-mediated membrane organization and movement of CLRs in the plasma membrane, which underlies cytoskeletal changes and cell migration.

\section{MATERIALS AND METHODS}

\section{Mice}

$\mathrm{Cd} 37^{-/-}$mice (male, average age of 3 months) were generated by homologous recombination (Knobeloch et al., 2000) and fully backcrossed to the C57BL/6J background (van Spriel et al., 2004). $C d 37^{+/+}$(WT) littermates were matched for age and sex. $C d 37^{+/+}$and $\mathrm{Cd} 37^{-/}$mice were bred in the Central Animal Laboratory of Radboud University Medical Center. CD11 $\mathrm{c}^{\Delta \mathrm{CLEC}-2}$ mice (C57BL/6J background), selectively lacking CLEC-2 in CD11 $\mathrm{c}^{+}$(also known as Itgax ${ }^{+}$) cells, were generated by crossing $C d 11 c$-cre and $C l e c 1 b^{\mathrm{fl} / \mathrm{fl}}$ mice, as previously described (Acton et al., 2014). All murine studies complied with European legislation (directive 2010/63/EU of the European Commission) and were approved by local authorities (Centrale Commissie Dierproeven, The Netherlands, and the Institutional Animal Ethics Committee Review Board, Cancer Research UK and Home Office, UK) for the care and use of animals with related codes of practice. All mice were housed in top-filter cages and fed a standard diet with freely available water and food.

\section{Isolation of whole blood}

Mice were anesthetized with isoflurane and whole blood was harvested via retro-orbital punction and collected in a tube containing acid-citratedextrose mixture $[25 \mathrm{~g} / 1$ sodium citrate, $20 \mathrm{~g} / 1$ glucose (both from SigmaAldrich) and $15 \mathrm{~g} / 1$ citric acid (Merck)] to prevent clotting.

\section{Cell culture}

BMDCs were generated by culturing total murine bone marrow cells in complete medium [RPMI 1640 medium (Gibco, via Thermo Fisher Scientific), $10 \%$ fetal calf serum (FCS; Greiner Bio-One), $1 \%$ UltraGlutamine-I (UG; Lonza), 1\% antibiotic-antimycotic (AA; Gibco, via Thermo Fisher Scientific) and $50 \mu \mathrm{M} \beta$-mercapto-ethanol (SigmaAldrich)], containing $20 \mathrm{ng} / \mathrm{ml}$ murine granulocyte-macrophage colonystimulating factor (mGM-CSF; Peprotech, via Bio-Connect), as adapted from previously described protocols (Lutz et al., 1999). On day 6, BMDCs were additionally stimulated with $10 \mathrm{ng} / \mathrm{ml}$ LPS (Sigma-Aldrich) for $24 \mathrm{~h}$, unless stated otherwise.

Primary human dermal LECs were purchased from PromoCell and cultured in the manufacturer's recommended medium (Endothelial Cell Growth Medium MV2, PromoCell) supplemented with $35 \mu \mathrm{g} / \mathrm{ml}$ gentamicin (Gibco, via Fisher Scientific). As previously described (Ahmed et al., 2011), LECs were dissociated using a 2:1 ratio of trypsin $(2.5 \mathrm{mg} / \mathrm{ml})$ to EDTA $(0.02 \%)$ (both from Sigma-Aldrich) and seeded onto 12 -well tissue culture plates coated with $2 \%$ gelatin (Sigma-Aldrich). Seeding density was chosen to yield a confluent LEC monolayer within 24 h. TNF $\alpha$ ( $100 \mathrm{U} / \mathrm{ml}$; R\&D Systems, via Bio-Techne Ltd) was added to confluent LEC monolayers for $24 \mathrm{~h}$ before analysing static adhesion and migration of DCs (Johnson and Jackson, 2013; Maddaluno et al., 2009; Podgrabinska et al., 2009).

RAW264.7 murine macrophages (RAW; origin American Type Culture Collection) were cultured in RPMI 1640 medium (Gibco, via Thermo Fisher Scientific) supplemented with 10\% FCS (Greiner Bio-One), 1\% UG (Lonza) and 1\% AA (Gibco, via Thermo Fisher Scientific). RAW cells were mycoplasma-free. The HEK-293T (HEK-293 cells expressing the large T-antigen of simian virus 40) cell line (Brummer et al., 2018; Haining et al., 2012, 2017; Noy et al., 2016; Reyat et al., 2017) was cultured in complete Dulbecco's modified Eagle medium (DMEM) (Sigma-Aldrich) containing $10 \%$ FCS (Gibco, via Thermo Fisher Scientific), 4 mM L-glutamine, $100 \mathrm{U} / \mathrm{ml}$ penicillin and $100 \mu \mathrm{g} / \mathrm{ml}$ streptomycin (Thermo Fisher Scientific). HEK$293 \mathrm{~T}$ cells were mycoplasma-free, genomic sequencing has recently confirmed their origin as human and resistance to neomycin is consistent with their continued expression of the large $\mathrm{T}$ antigen.

FRCs (Acton et al., 2014) were cultured in high-glucose DMEM with GlutaMAX supplement (Gibco, via Thermo Fisher Scientific) supplemented with $10 \%$ FCS (Sigma-Aldrich), $1 \%$ penicillin-streptomycin and $1 \%$ insulintransferrin-selenium (both Gibco, via Thermo Fisher Scientific) at $37^{\circ} \mathrm{C}$, $10 \% \mathrm{CO}_{2}$, and split using cell-dissociation buffer (Gibco, via Thermo Fisher Scientific). FRCs have been regularly analysed by flow cytometry for authentication, and were screened by the Cell Services Department at the Francis Crick Institute (London, UK) to rule out contamination.

\section{Constructs and transfection}

mCD37-pmCherry was generated by fluorescent protein swap of GFP from mCD37-pEGFP (Meyer-Wentrup et al., 2007) with mCherry from pmCherry-N1 (Clontech, via Takara Bio Europe) using AgeI and BsrGI restriction sites (New England Biolabs, via Bioké). RAW macrophages $\left(5 \times 10^{5}\right.$ cells/transfection) were transfected with $0.5 \mu \mathrm{g}$ mCD37-mCherry and/or $0.5 \mu \mathrm{g}$ pAcGFP-mCLEC-2 as previously described (Pollitt et al., 2014) using FuGENE HD according to the manufacturer's instructions (Promega).

Human (h)CLEC-2-MYC construct with C-terminal MYC tags (Fuller et al., 2007) was generated in the pEF6 expression vector (Invitrogen, via Thermo Fisher Scientific). Human (h)FLAG-CLEC-2 construct was kindly provided by Prof. S. P. Watson (Hughes et al., 2010). The FLAG-human CD37 and other human tetraspanin constructs were produced using the 
pEF6 expression vector with an N-terminal FLAG tag, as described previously (Protty et al., 2009). HEK-293T cells were transiently transfected with hCLEC-2-MYC and FLAG epitope-tagged human tetraspanin expression constructs using polyethylenimine (Sigma-Aldrich) as described previously (Ehrhardt et al., 2006; Noy et al., 2015).

\section{Immunoprecipitation and western blotting}

Transfected HEK-293T cells were lysed in 1\% digitonin (Acros Organics, via Thermo Fisher Scientific) and immunoprecipitated with mouse antiFLAG antibody (clone M2, cat. no. F1804, $1 \mu \mathrm{g}$ per immunoprecipitate; Sigma-Aldrich) as described previously (Haining et al., 2012). Western blots were stained with mouse anti-MYC (clone 9B11, cat. no. 2276, batch no. 11/2016, $0.2 \mu \mathrm{g} / \mathrm{ml}$; Cell Signaling Technology, via New England Biolabs) or rabbit anti-FLAG (polyclonal, cat. no. F7425, $0.2 \mu \mathrm{g} / \mathrm{ml}$; Sigma-Aldrich) antibodies, followed by IRDye 680RD- or $800 \mathrm{CW}$ conjugated secondary antibodies (LI-COR Biotechnology), and imaged using an Odyssey Infrared Imaging System (LI-COR Biotechnology).

\section{Flow cytometry}

Murine whole blood was incubated in phosphate-buffered saline (PBS; Braun) in the presence of $1 \mathrm{mM}$ EDTA (Amresco, via VWR International) to prevent platelet aggregation, and 2\% normal goat serum (NGS; SigmaAldrich) to block Fc receptors for $15 \mathrm{~min}$ at $4^{\circ} \mathrm{C}$. Next, murine blood cells were stained with anti-mouse CLEC-2 (clone INU1, $10 \mu \mathrm{g} / \mathrm{ml}$; May et al., 2009) or an appropriate isotype control, and subsequently with goat-anti-rat IgG-APC (cat. no. 551019, batch no. 7118669, 1:200 dilution; BD Biosciences). To discriminate blood platelets, staining with anti-mouse CD41-PE (clone MWReg30, cat. no. 558040, batch no. 46107, 1:25 dilution; BD Biosciences) was performed.

Splenocytes or cell lines were stained for $30 \mathrm{~min}$ at $4{ }^{\circ} \mathrm{C}$ in PBS (Braun) containing $1 \%$ bovine serum albumin (BSA; Roche) and $0.05 \% \mathrm{NaN}_{3}$, and supplemented with $2 \%$ NGS (Sigma-Aldrich), with the following primary anti-mouse antibodies: CLEC-2 (clone INU1, $10 \mu \mathrm{g} / \mathrm{ml}$; May et al., 2009), CD37 (Fig. S5A; clone Duno85, cat. no. 146202, batch no. B170846, 1:50 dilution; BioLegend), B220-FITC (CD45R, clone RA3-6B2, cat. no. 103206, batch no. B161286, 1:100 dilution; BioLegend), CD11c-Alexa Fluor 488 (clone N418, cat. no. 557400, batch no. 4078759, 1:50 dilution; BioLegend), NK1.1-PE (clone PK136, cat. no. 553165, batch no. 2209691, 1:50 dilution; BD Biosciences), GR1-PE (clone RB6-8C5, cat. no. 108408, batch no. B116829, 1:50 dilution; BioLegend), CD11b-PerCP (clone M1/70, cat. no. 101230, batch no. B172190, 1:200 dilution; BioLegend), CD3-biotin (CD3e, clone 145-2C11, cat. no. 13-0031-85, batch no. E019673, 1:100 dilution; eBioscience, via Thermo Fisher Scientific), or appropriate isotype controls. This was followed by incubation with streptavidin-PerCP (cat. no. 405213, batch no. B190408, 1:250 dilution; BioLegend) or goat anti-rat IgG Alexa Fluor 647 (cat. no. A21247, batch no. 1611119, 1:400 dilution; Invitrogen, via Thermo Fisher Scientific). CD37 in transfected HEK-293T cells was detected using a fluorescein isothiocyanate (FITC)-conjugated mouse anti-human CD37 antibody (cat. no. MCA483F, batch no. 1098, $10 \mu \mathrm{g} / \mathrm{ml}$; Serotec). Stained cells were analysed using a FACSCalibur (BD Biosciences) or CyanADP (Beckman Coulter) flow cytometer, and FlowJo software (TreeStar).

\section{Rhodocytin stimulation and cytokine production}

Single-cell suspensions of splenocytes were generated by passing spleens through a $100 \mu \mathrm{m}$ cell strainer (Falcon, via Corning). To lyse erythrocytes, splenocytes were treated with ACK lysis buffer $\left[0.15 \mathrm{M} \mathrm{NH}_{4} \mathrm{Cl}, 10 \mathrm{mM}\right.$ $\mathrm{KHCO}_{3}$ (both from Merck), $0.1 \mathrm{mM}$ EDTA (Sigma-Aldrich); $\mathrm{pH}$ 7.3] for $2 \mathrm{~min}$ on ice. Splenocytes $\left(5 \times 10^{5}\right.$ cells) were stimulated with either $15 \mu \mathrm{g}$ rhodocytin [purified as previously described (Eble et al., 2001)], PMA/ ionomycin $(100 \mathrm{ng} / \mathrm{ml}$ and $500 \mathrm{ng} / \mathrm{ml}$, both from Sigma-Aldrich), or with complete RPMI 1640 medium (unstimulated), and incubated overnight at $37^{\circ} \mathrm{C}, 5 \% \mathrm{CO}_{2}$. To measure IL- 6 levels in the supernatant of stimulated cell cultures, NUNC Maxisorp 96-well plates (eBioscience, via Thermo Fisher Scientific) were coated with capture anti-mouse IL-6 antibody (clone MP520F3, cat. no. 504506, batch. no. B111147, $2 \mu \mathrm{g} / \mathrm{ml}$; BD Biosciences) in
$0.1 \mathrm{M}$ carbonate buffer ( $\mathrm{pH} 9.6$ ) overnight at $4^{\circ} \mathrm{C}$. Wells were blocked with PBS (Braun) containing 1\% BSA (Roche) and 1\% FCS (Greiner Bio-One) for $1 \mathrm{~h}$ at room temperature (RT), washed, and incubated with $50 \mu 1$ of sample and standard (2-fold serial dilutions starting from 10,000 pg/ml) (eBioscience, via Thermo Fisher Scientific). After $2 \mathrm{~h}$ incubation at RT, wells were incubated with biotinylated anti-mouse IL-6 (MP5-32C11, cat. no. 554402, batch no. B145730, $1 \mu \mathrm{g} / \mathrm{ml}$, BD Biosciences) for $1 \mathrm{~h}$ at RT, followed by incubation with horseradish peroxidase-conjugated streptavidin (cat. no. 00-4100-94, batch no. 4298336, 1:5000 dilution, Invitrogen, via Thermo Fisher Scientific) for $30 \mathrm{~min}$ at RT. Complexes were visualized using TMB substrate (Sigma-Aldrich), and reactions were stopped by adding $0.8 \mathrm{M} \mathrm{H}_{2} \mathrm{SO}_{4}$ (Merck Millipore). Absorbance was measured at $450 \mathrm{~nm}$ using an iMark plate reader (Bio-Rad).

\section{Static adhesion and migration assay}

Adhesion was assessed by direct microscopic observation as previously described (Butler et al., 2005). LECs in 12-well plates were washed three times with Medium 199 supplemented with $0.15 \%$ BSA Fraction V $7.5 \%$ (M199BSA, both from Gibco, via Thermo Fisher Scientific) to remove residual cytokines. BMDCs $\left(1 \times 10^{6}\right)$ were added on top of the LEC monolayer and incubated for $10 \mathrm{~min}$ at $37^{\circ} \mathrm{C}, 5 \% \mathrm{CO}_{2}$. Nonadherent cells were removed from the LECs by gently washing three times with M199BSA. Imaging was performed using an Olympus Invert X70 microscope enclosed at $37^{\circ} \mathrm{C}$. Digital recordings of five fields of view of the LEC surface were made using phase-contrast microscopy immediately and $10 \mathrm{~min}$ after washing away nonadherent cells. In between, time-lapse imaging (one image every $10 \mathrm{~s}$ ) was performed for $5 \mathrm{~min}$ of one field of view to assess cell migration at $37^{\circ} \mathrm{C}$. Digitized recordings were analysed offline using Image-Pro software (version 6.2, DataCell). The numbers of adherent cells were counted in the video fields, averaged, converted to cells per $\mathrm{mm}^{2}$ using the calibrated microscope field dimensions, and multiplied by the known surface area of the well to calculate the total number of adherent cells. This number was divided by the known total number of cells added to obtain the percentage of the cells that had adhered. Cell tracks of live cells were analysed using the Manual Tracking plugin in Fiji/ImageJ software. Migration velocity was calculated as the length of each cell path per time $(\mu \mathrm{m} / \mathrm{min})$, and the $x y$ trajectories were converted into MSD (in $\mu \mathrm{m}^{2}$ ) as previously described (van Rijn et al., 2016).

\section{D protrusion assay and DC-FRC co-cultures}

FRCs $\left(0.7 \times 10^{4}\right)$ were seeded on glass-bottomed cell culture plates (MatTek). LPS-activated BMDCs $\left(0.3 \times 10^{6}\right)$ were seeded onto 3D collagen (type I, rat tail)/Matrigel matrix (both from Corning, via Thermo Fisher Scientific) supplemented with $10 \%$ minimum essential medium alpha medium (MEMalpha, Invitrogen, via Thermo Fisher Scientific) and $10 \%$ FCS (Greiner Bio-One), either alone on glass-bottomed cell culture plates (MatTek) or on top of the FRCs $24 \mathrm{~h}$ after seeding. For CLEC-2 activation, $20 \mu \mathrm{g} / \mathrm{ml}$ recombinant podoplanin-Fc (rPDPN-Fc; Sino Biological) (Acton et al., 2012) was added to the gel as all components were mixed. Gels were incubated overnight at $37^{\circ} \mathrm{C}, 5 \% \mathrm{CO}_{2}$. The next day, cultures were fixed with DiaPath Antigenfix (Solmedia) for $3 \mathrm{~h}$ at RT, followed by permeabilization and blocking with $2 \%$ BSA (Roche), $1 \%$ normal mouse serum and $0.2 \%$ Triton X-100 in PBS for $2 \mathrm{~h}$ at RT before staining. F-actin and cell nuclei were visualized using TRITC-phalloidin (cat. no. P1951-1MG) and 4',6-diamidino-2-phenylindole (DAPI; cat. no. D9542-1MG), respectively (both 1:500 dilution, both from Invitrogen, via Thermo Fisher Scientific). Podoplanin was stained using a hamster antimouse podoplanin antibody (Fig. S5B; clone 8.1.1., batch no. 201572, 1:500 dilution, Acris Antibodies) followed by secondary goat anti-hamster Alexa Fluor 647 antibody (cat. no. A-21451, batch no. 1812653, dilution 1:100, Invitrogen, via Thermo Fisher Scientific). Cells were imaged on a Leica SP5 confocal microscope, and analysed using Fiji/ImageJ software. Z stacks of $120 \mu \mathrm{m}(10 \mu \mathrm{m} / \mathrm{step})$ were projected with ImageJ Z Project (maximum projection), and the number and length of protrusions were measured. Cell morphology (=perimeter ${ }^{2} / 4 \pi$ area) was calculated using the area and perimeter of cells by manually drawing around the cell shape using F-actin staining. 


\section{Microcontact printing}

Poly(dimethylsiloxane) (PDMS) stamps with a regular pattern of $5 \mu \mathrm{m}$ circular spots were prepared as previously described (Van Den Dries et al., 2012). Stamps were incubated with $20 \mu \mathrm{g} / \mathrm{ml} \mathrm{rPDPN-Fc} \mathrm{(cat.} \mathrm{no.}$ 50256-M03H-100 $\mu \mathrm{g}$, batch no. LC05NO2305; Sino Biological) for CLEC-2 stimulation, and $10 \mu \mathrm{g} / \mathrm{ml}$ donkey anti-rabbit IgG (H\&L)-Alexa Fluor 647 (cat. no. A31573, batch no. 1826679; Invitrogen, via Thermo Fisher Scientific) to visualize the spots, diluted in PBS (Braun) for $1 \mathrm{~h}$ at RT. Stamps were washed with demineralized water and dried under a nitrogen stream. The stamp was applied to a cleaned glass coverslip for 1 min and subsequently removed. Transfected RAW macrophages were seeded on the stamped area and incubated for $12 \mathrm{~min}$ at $37^{\circ} \mathrm{C}$. Cells were fixed with $4 \%$ paraformaldehyde (Merck) for $20 \mathrm{~min}$ at RT. Samples were washed with PBS (Braun) and demineralized water (MilliQ; Merck Millipore) and embedded in Mowiol (Sigma-Aldrich). Imaging was performed on an epi-fluorescence Leica DMI6000 microscope, and plot profiles were created using Fiji/ImageJ software. For the population of cells in contact with rPDPN-Fc spots, we determined the percentage showing on-stamp enrichment of mCLEC-2 GFP by independent visual analysis with support of Fiji/ImageJ software.

\section{Statistics}

Statistical differences between two groups (e.g. WT and $C d 37^{-/-}$cells) regarding IL-6 production, adhesion, CLEC-2 enrichment and NFATluciferase activity were determined using (un)paired Student's $t$-test or nonparametric Mann-Whitney test (in the case of non-Gaussian distribution). Statistical differences between three groups (e.g. WT, $C d 37^{-/-}$and CD11 $\mathrm{c}^{\Delta \mathrm{CLEC}-2}$ cells) or two or more parameters (e.g. genotype and time) were determined using one-way ANOVA with Dunn's multiple comparisons test, or two-way ANOVA with Sidak's or Tukey's multiple comparisons test, respectively. Statistical tests were performed using GraphPad Prism software, and all differences were considered to be statistically significant at $P \leq 0.05$.

\section{Acknowledgements}

We thank Prof. Steve P. Watson [University of Birmingham, UK; British Heart Foundation Chair (CH03/03)] and Dr Mark Wright (Monash University, Melbourne, Australia) for discussion and critical reading of the manuscript. We also thank Jing Yang for her contribution to the research during her role as research assistant in the laboratory of M.G.T.

Competing interests

The authors declare no competing or financial interests.

\section{Author contributions}

Conceptualization: C.M.d.W., S.v.D., M.G.T., S.E.A., A.B.v.S.; Methodology: C.M.d.W., M.G.T., S.E.A., A.B.v.S.; Validation: C.M.d.W., A.L.M.; Formal analysis: C.M.d.W., A.L.M.; Investigation: C.M.d.W., A.L.M., A.v.d.S., N.D.T., E.J., H.M.M., S.E.A.; Resources: J.A.E., B.N., H.M.M., S.E.A.; Writing - original draft: C.M.d.W.; Writing - review \& editing: A.L.M., S.v.D., H.M.M., M.G.T., S.E.A., A.B.v.S.; Visualization: C.M.d.W., A.L.M.; Supervision: C.G.F., M.G.T., S.E.A., A.B.v.S. Funding acquisition: C.M.d.W., A.L.M., H.M.M., C.G.F., M.G.T., S.E.A., A.B.v.S.

\section{Funding}

This work was supported by Erasmus+ [Staff Mobility Grant to C.M.d.W.] Nederlandse Organisatie voor Wetenschappelijk Onderzoek [019.162LW.004 to C.M.d.W.; Scientific Research Spinoza Award to C.G.F.; 864.11 .006 and ICI-024.002.009 to A.B.v.S.], the Biotechnology and Biological Sciences Research Council [PhD Studentship to A.L.M.], the Medical Research Council [PhD Studentship to N.D.T.; G0400247 to M.G.T.; MC_U12266B to S.E.A.], Deutsche Forschungsgemeinschaft [SFB1009 A09 to J.A.E.; SFB/TR 240 to B.N.], Arthritis Research UK [19899 to H.M.M.], the European Research Council [269019 to C.G.F.; 724281 to A.B.v.S.], KWF Kankerbestrijding [KUN2009-4402 to C.G.F.; KUN2014-6845 to A.B.v.S.], the British Heart Foundation [FS/08/062/25797 to M.G.T.] and Cancer Research UK [CRUK-A19763 to S.E.A.]

\section{Supplementary information}

Supplementary information available online at

http://jcs.biologists.org/lookup/doi/10.1242/jcs.214551.supplemental

\section{References}

Acton, S. E., Astarita, J. L., Malhotra, D., Lukacs-Kornek, V., Franz, B., Hess P. R., Jakus, Z., Kuligowski, M., Fletcher, A. L., Elpek, K. G. et al. (2012) Podoplanin-rich stromal networks induce dendritic cell motility via activation of the C-type lectin receptor CLEC-2. Immunity 37, 276-289.

Acton, S. E., Farrugia, A. J., Astarita, J. L., Mourão-Sá, D., Jenkins, R. P., Nye, E., Hooper, S., van Blijswijk, J., Rogers, N. C., Snelgrove, K. J. et al. (2014) Dendritic cells control fibroblastic reticular network tension and lymph node expansion. Nature 514, 498-502.

Ahmed, S. R., McGettrick, H. M., Yates, C. M., Buckley, C. D., Ratcliffe, M. J., Nash, G. B. and Rainger, G. E. (2011). Prostaglandin D2 regulates CD4+ memory $T$ cell trafficking across blood vascular endothelium and primes these cells for clearance across lymphatic endothelium. J. Immunol. 187, 1432-1439.

Astarita, J. L., Cremasco, V., Fu, J., Darnell, M. C., Peck, J. R., Nieves-Bonilla, J. M., Song, K., Kondo, Y., Woodruff, M. C., Gogineni, A. et al. (2015). The CLEC-2-podoplanin axis controls the contractility of fibroblastic reticular cells and lymph node microarchitecture. Nat. Immunol. 16, 75-84.

Bertozzi, C. C., Schmaier, A. A., Mericko, P., Hess, P. R., Zou, Z., Chen, M. Chen, C.-Y., Xu, B., Lu, M., Zhou, D. et al. (2010). Platelets regulate lymphatic vascular development through CLEC-2-SLP-76 signaling. Blood 116, 661-670.

Blank, N., Schiller, M., Krienke, S., Wabnitz, G., Ho, A. D. and Lorenz, H.-M. (2007). Cholera toxin binds to lipid rafts but has a limited specificity for ganglioside GM1. Immunol. Cell Biol. 85, 378-382.

Brown, G. D. G. and Gordon, S. (2001). Immune recognition. A new receptor for beta-glucans. Nature 413, 36-37.

Brummer, T., Pigoni, M., Rossello, A., Wang, H., Noy, P. J., Tomlinson, M. G. Blobel, C. P. and Lichtenthaler, S. F. (2018). The metalloprotease ADAM10 (a disintegrin and metalloprotease 10) undergoes rapid, postlysis autocatalytic degradation. FASEB J. 32, 3560-3573.

Butler, L. M., Rainger, G. E., Rahman, M. and Nash, G. B. (2005). Prolonged culture of endothelial cells and deposition of basement membrane modify the recruitment of neutrophils. Exp. Cell Res. 310, 22-32.

Cambi, A., De Lange, F., Van Maarseveen, N. M., Nijhuis, M., Joosten, B. Van Dijk, E. M. H. P., De Bakker, B. I., Fransen, J. A. M., Bovee-Geurts, P. H. M., Van Leeuwen, F. N. et al. (2004). Microdomains of the C-type lectin DC-SIGN are portals for virus entry into dendritic cells. J. Cell Biol. 164, 145-155.

Carmo, A. M. and Wright, M. D. (1995). Association of the transmembrane 4 superfamily molecule CD53 with a tyrosine phosphatase activity. Eur. J. Immunol. 25, 2090-2095.

Chang, C.-H., Chung, C.-H., Hsu, C.-C., Huang, T.-Y. and Huang, T.-F. (2010). A novel mechanism of cytokine release in phagocytes induced by aggretin, a snake venom C-type lectin protein, through CLEC-2 ligation. J. Thromb. Haemost. 8 , 2563-2570.

Charrin, S., Le Naour, F., Oualid, M., Billard, M., Faure, G., Hanash, S. M. Boucheix, C. and Rubinstein, E. (2001). The major CD9 and CD81 molecular partner. Identification and characterization of the complexes. J. Biol. Chem. 276 14329-14337.

Charrin, S., le Naour, F., Silvie, O., Milhiet, P.-E. E., Boucheix, C. and Rubinstein, E. (2009). Lateral organization of membrane proteins: tetraspanins spin their web. Biochem. J. 420, 133-154.

Chattopadhyay, N., Wang, Z., Ashman, L. K., Brady-Kalnay, S. M. and Kreidberg, J. A. (2003). alpha3beta1 integrin-CD151, a component of the cadherin-catenin complex, regulates PTP $\mu$ expression and cell-cell adhesion. J. Cell Biol. 163, 1351-1362

Claas, C., Stipp, C. S. and Hemler, M. E. (2001). Evaluation of prototype transmembrane 4 superfamily protein complexes and their relation to lipid rafts J. Biol. Chem. 276, 7974-7984.

Colonna, M., Samaridis, J. and Angman, L. (2000). Molecular characterization of two novel C-type lectin-like receptors, one of which is selectively expressed in human dendritic cells. Eur. J. Immunol. 30, 697-704.

De Turris, V., Teloni, R., Chiani, P., Bromuro, C., Mariotti, S., Pardini, M., Nisini, R., Torosantucci, A. and Gagliardi, M. C. (2015). Candida albicans targets a lipid raft/dectin-1 platform to enter human monocytes and induce antigen specific T cell responses. PLOS ONE 10, e0142531.

de Winde, C. M., Zuidscherwoude, M., Vasaturo, A., van der Schaaf, A., Figdor C. G. and van Spriel, A. B. (2015). Multispectral imaging reveals the tissue distribution of tetraspanins in human lymphoid organs. Histochem. Cell Biol. 144 133-146.

de Winde, C. M., Veenbergen, S., Young, K. H., Xu-Monette, Z. Y., Wang, X.-X Xia, Y., Jabbar, K. J., Van Den Brand, M., Van Der Schaaf, A., Elfrink, S. et al. (2016). Tetraspanin CD37 protects against the development of B cell lymphoma. J. Clin. Invest. 126, 653-666.

Dornier, E., Coumailleau, F., Ottavi, J.-F., Moretti, J., Boucheix, C., Mauduit, P., Schweisguth, F. and Rubinstein, E. (2012). Tspanc8 tetraspanins regulate ADAM10/Kuzbanian trafficking and promote Notch activation in flies and mammals. J. Cell Biol. 199, 481-496.

Eble, J. A., Beermann, B., Hinz, H.-J. and Schmidt-Hederich, A. (2001). $\alpha 2 \beta 1$ integrin is not recognized by rhodocytin but is the specific, high affinity target of rhodocetin, an RGD-independent disintegrin and potent inhibitor of cell adhesion to collagen. J. Biol. Chem. 276, 12274-12284. 
Ehrhardt, C., Schmolke, M., Matzke, A., Knoblauch, A., Will, C., Wixler, V. and Ludwig, S. (2006). Polyethylenimine, a cost-effective transfection reagent. Signal. Transduct. 6, 179-184.

Figdor, C. G. and van Spriel, A. B. (2009). Fungal pattern-recognition receptors and tetraspanins: partners on antigen-presenting cells. Trends Immunol. 31, 91-96.

Fuller, G. L. J., Williams, J. A. E., Tomlinson, M. G., Eble, J. A., Hanna, S. L., Pöhlmann, S., Suzuki-Inoue, K., Ozaki, Y., Watson, S. P. and Pearce, A. C. (2007). The C-type lectin receptors CLEC-2 and Dectin-1, but not DC-SIGN signal via a novel YXXL-dependent signaling cascade. J. Biol. Chem. 282 12397-12409.

Gartlan, K. H., Wee, J. L., Demaria, M. C., Nastovska, R., Chang, T. M., Jones, E. L., Apostolopoulos, V., Pietersz, G. A., Hickey, M. J., van Spriel, A. B. et al. (2013). Tetraspanin CD37 contributes to the initiation of cellular immunity by promoting dendritic cell migration. Eur. J. Immunol. 43, 1208-1219.

Goodridge, H. S., Reyes, C. N., Becker, C. A., Katsumoto, T. R., Ma, J., Wolf, A. J., Bose, N., Chan, A. S. H., Magee, A. S., Danielson, M. E. et al. (2011) Activation of the innate immune receptor Dectin-1 upon formation of a "phagocytic synapse". Nature 472, 471-475

Haining, E. J., Yang, J., Bailey, R. L., Khan, K., Collier, R., Tsai, S., Watson, S. P., Frampton, J., Garcia, P. and Tomlinson, M. G. (2012). The TspanC8 subgroup of tetraspanins interacts with A disintegrin and metalloprotease 10 (ADAM10) and regulates its maturation and cell surface expression. J. Biol. Chem. 287 39753-39765.

Haining, E. J., Matthews, A. L., Noy, P. J., Romanska, H. M., Harris, H. J., Pike, J., Morowski, M., Gavin, R. L., Yang, J., Milhiet, P.-E. et al. (2017). Tetraspanin Tspan9 regulates platelet collagen receptor GPVI lateral diffusion and activation. Platelets 28, 629-642.

Hemler, M. E. (2005). Tetraspanin functions and associated microdomains. Nat Rev. Mol. Cell Biol. 6, 801-811.

Hughes, C. E., Pollitt, A. Y., Mori, J., Eble, J. A., Tomlinson, M. G., Hartwig, J. H., O'Callaghan, C. A., Fütterer, K. and Watson, S. P. (2010). CLEC-2 activates Syk through dimerization. Blood 115, 2947-2955.

Johnson, L. A. and Jackson, D. G. (2013). The chemokine CX3CL1 promotes trafficking of dendritic cells through inflamed lymphatics. J. Cell Sci. 126 5259-5270

Jones, E. L., Wee, J. L., Demaria, M. C., Blakeley, J., Ho, P. K., Vega-Ramos, J., Villadangos, J. A., van Spriel, A. B., Hickey, M. J., Hämmerling, G. J. et al. (2016). Dendritic cell migration and antigen presentation are coordinated by the opposing functions of the tetraspanins CD82 and CD37. J. Immunol. 196, 978-987.

Kerrigan, A. M., Dennehy, K. M., Mourão-Sá, D., Faro-Trindade, I., Willment J. A., Taylor, P. R., Eble, J. A., Reis e Sousa, C. and Brown, G. D. (2009) CLEC-2 is a phagocytic activation receptor expressed on murine peripheral blood neutrophils. J. Immunol. 182, 4150-4157.

Knobeloch, K.-P., Wright, M. D., Ochsenbein, A. F., Liesenfeld, O., Löhler, J., Zinkernagel, R. M., Horak, I. and Orinska, Z. (2000). Targeted inactivation of the tetraspanin CD37 impairs T-cell-dependent B-cell response under suboptimal costimulatory conditions. Mol. Cell. Biol. 20, 5363-5369.

Lapalombella, R., Yeh, Y.-Y., Wang, L., Ramanunni, A., Rafiq, S., Jha, S., Staubli, J., Lucas, D. M., Mani, R., Herman, S. E. M. et al. (2012). Tetraspanin CD37 directly mediates transduction of survival and apoptotic signals. Cancer Cell 21, 694-708

Levy, S. and Shoham, T. (2005). The tetraspanin web modulates immunesignalling complexes. Nat. Rev. Immunol. 5, 136-148.

Lowe, K. L., Navarro-Nuñez, L., Bénézech, C., Nayar, S., Kingston, B. L., Nieswandt, B., Barone, F., Watson, S. P., Buckley, C. D. and Desanti, G. E. (2015). The expression of mouse CLEC-2 on leucocyte subsets varies according to their anatomical location and inflammatory state. Eur. J. Immunol. 45 2484-2493.

Lutz, M. B., Kukutsch, N., Ogilvie, A. L. J., Rößner, S., Koch, F., Romani, N. and Schuler, G. (1999). An advanced culture method for generating large quantities of highly pure dendritic cells from mouse bone marrow. J. Immunol. Methods 223, 77-92

Maddaluno, L., Verbrugge, S. E., Martinoli, C., Matteoli, G., Chiavelli, A., Zeng, Y., Williams, E. D., Rescigno, M. and Cavallaro, U. (2009). The adhesion molecule $\mathrm{L} 1$ regulates transendothelial migration and trafficking of dendritic cells. J. Exp. Med. 206, 623-635.

Manne, B. K., Badolia, R., Dangelmaier, C. A. and Kunapuli, S. P. (2015). C-type lectin like receptor 2 (CLEC-2) signals independently of lipid raft microdomains in platelets. Biochem. Pharmacol. 93, 163-170.

Mantegazza, A. R., Barrio, M. M., Moutel, S., Bover, L., Weck, M., Brossart, P., Teillaud, J. L. and Mordoh, J. (2004). CD63 tetraspanin slows down cell migration and translocates to the endosomal-lysosomal-MIICs route after extracellular stimuli in human immature dendritic cells. Blood 104, 1183-1190.

May, F., Hagedorn, I., Pleines, I., Bender, M., Vögtle, T., Eble, J., Elvers, M. and Nieswandt, B. (2009). CLEC-2 is an essential platelet-activating receptor in hemostasis and thrombosis. Blood 114, 3464-3472.
Meyer-Wentrup, F., Figdor, C. G., Ansems, M., Brossart, P., Wright, M. D., Adema, G. J. and van Spriel, A. B. (2007). Dectin-1 interaction with tetraspanin CD37 inhibits IL-6 production. J. Immunol. 178, 154-162.

Mócsai, A., Ruland, J. and Tybulewicz, V. L. J. (2010). The SYK tyrosine kinase: a crucial player in diverse biological functions. Nat. Rev. Immunol. 10, 387-402.

Mourão-Sá, D., Robinson, M. J., Zelenay, S., Sancho, D., Chakravarty, P., Larsen, R., Plantinga, M., Van Rooijen, N., Soares, M. P., Lambrecht, B. et al. (2011). CLEC-2 signaling via Syk in myeloid cells can regulate inflammatory responses. Eur. J. Immunol. 41, 3040-3053.

Nobes, C. D. and Hall, A. (1995). Rho, Rac, and Cdc42 GTPases regulate the assembly of multimolecular focal complexes associated with actin stress fibers, lamellipodia, and filopodia. Cell 81, 53-62.

Noy, P. J., Lodhia, P., Khan, K., Zhuang, X., Ward, D. G., Verissimo, A. R., Bacon, A. and Bicknell, R. (2015). Blocking CLEC14A-MMRN2 binding inhibits sprouting angiogenesis and tumour growth. Oncogene 34, 5821-5831.

Noy, P. J., Yang, J., Reyat, J. S., Matthews, A. L., Charlton, A. E., Furmston, J. Rogers, D. A., Rainger, G. E. and Tomlinson, M. G. (2016). TspanC 8 tetraspanins and A Disintegrin and Metalloprotease 10 (ADAM10) interact via their extracellular regions: evidence for distinct binding mechanisms for different TspanC8 proteins. J. Biol. Chem. 291, 3145-3157.

Olson, M. F. and Sahai, E. (2009). The actin cytoskeleton in cancer cell motility. Clin. Exp. Metastasis 26, 273-287.

Parri, M. and Chiarugi, P. (2010). Rac and Rho GTPases in cancer cell motility control. Cell Commun. Signal. 8, 23.

Podgrabinska, S., Kamalu, O., Mayer, L., Shimaoka, M., Snoeck, H., Randolph, G. J. and Skobe, M. (2009). Inflamed lymphatic endothelium suppresses dendritic cell maturation and function via Mac-1/ICAM-1-dependent mechanism. J. Immunol. 183, 1767-1779.

Pollitt, A. Y., Grygielska, B., Leblond, B., Désiré, L., Eble, J. A. and Watson, S. P (2010). Phosphorylation of CLEC-2 is dependent on lipid rafts, actin polymerization, secondary mediators, and Rac. Blood 115, 2938-2946.

Pollitt, A. Y., Poulter, N. S., Gitz, E., Navarro-nuñez, L., Wang, Y.-J., Hughes C. E., Thomas, S. G., Nieswandt, B., Douglas, M. R., Owen, D. M., et al. (2014) Syk and Src family kinases regulate C-type lectin receptor 2 (CLEC-2)-mediated clustering of podoplanin and platelet adhesion to lymphatic endothelial cells. J. Biol. Chem. 289, 35695-35710.

Protty, M. B., Watkins, N. A., Colombo, D., Thomas, S. G., Heath, V. L., Herbert, J. M. J., Bicknell, R., Senis, Y. A., Ashman, L. K., Berditchevski, F. et al. (2009). Identification of Tspan9 as a novel platelet tetraspanin and the collagen receptor GPVI as a component of tetraspanin microdomains. Biochem. J. 417, 391-401.

Reyat, J. S., Chimen, M., Noy, P. J., Szyroka, J., Rainger, G. E. and Tomlinson, M. G. (2017). ADAM10-interacting tetraspanins Tspan5 and Tspan17 regulate VE-cadherin expression and promote T lymphocyte transmigration. J. Immunol. 199, 666-676.

Serru, V., Le Naour, F., Billard, M., Azorsa, D. O., Lanza, F., Boucheix, C. and Rubinstein, E. (1999). Selective tetraspan-integrin complexes (CD81/ alpha4beta1, CD151/alpha3beta1, CD151/alpha6beta1) under conditions disrupting tetraspan interactions. Biochem. J. 340, 103-111.

Suzuki-Inoue, K., Fuller, G. L. J., García, A., Eble, J. A., Pöhlmann, S., Inoue, O Gartner, T. K., Hughan, S. C., Pearce, A. C., Laing, G. D. et al. (2006). A nove Syk-dependent mechanism of platelet activation by the C-type lectin receptor CLEC-2. Blood 107, 542-549.

Suzuki-Inoue, K., Inoue, O., Ding, G., Nishimura, S., Hokamura, K., Eto, K. Kashiwagi, H., Tomiyama, Y., Yatomi, Y., Umemura, K. et al. (2010). Essential in vivo roles of the C-type lectin receptor CLEC-2: embryonic/neonatal lethality of CLEC-2-deficient mice by blood/lymphatic misconnections and impaired thrombus formation of CLEC-2-deficient platelets. J. Biol. Chem. 285 , 24494-24507

Tarrant, J. M., Robb, L., van Spriel, A. B. and Wright, M. D. (2003). Tetraspanins: molecular organisers of the leukocyte surface. Trends Immunol. 24, 610-617.

Tejera, E., Rocha-Perugini, V., López-Martín, S., Pérez-Hernández, D., Bachir A. I., Horwitz, A. R., Vázquez, J., Sánchez-Madrid, F. and Yáñez-Mo, M. (2013) CD81 regulates cell migration through its association with Rac GTPase. Mol. Biol. Cell 24, 261-273

Van Den Dries, K., Van Helden, S. F. G., Te Riet, J., Diez-Ahedo, R., Manzo, C., Oud, M. H. M., Van Leeuwen, F. N., Brock, R., Garcia-Parajo, M. F., Cambi, A. et al. (2012). Geometry sensing by dendritic cells dictates spatial organization and PGE2-induced dissolution of podosomes. Cell. Mol. Life Sci. 69, 1889-1901.

van Deventer, S. J., Dunlock, V.-M. E. and van Spriel, A. B. (2017). Molecular interactions shaping the tetraspanin web. Biochem. Soc. Trans. 45, 741-750.

van Rijn, A., Paulis, L., Te Riet, J., Vasaturo, A., Reinieren-Beeren, I., van de Schaaf, A., Kuipers, A. J., Schulte, L. P., Jongbloets, B. C., Pasterkamp, R. J. et al. (2016). Semaphorin 7A promotes chemokine-driven dendritic cell migration. J. Immunol. 196, 459-468.

van Spriel, A. B., Puls, K. L., Sofi, M., Pouniotis, D., Hochrein, H., Orinska, Z. Knobeloch, K.-P. P., Plebanski, M. and Wright, M. D. (2004). A regulatory role for CD37 in T cell proliferation. J. Immunol. 172, 2953-2961.

van Spriel, A. B., de Keijzer, S., van der Schaaf, A., Gartlan, K. H., Sofi, M., Light A., Linssen, P. C., Boezeman, J. B., Zuidscherwoude, M., Reinieren-Beeren, 
I. et al. (2012). The tetraspanin CD37 orchestrates the $\alpha(4) \beta(1)$ integrin-Akt signaling axis and supports long-lived plasma cell survival. Sci. Signal. 5, ra82.

Worbs, T., Hammerschmidt, S. I. and Förster, R. (2017). Dendritic cell migration in health and disease. Nat. Rev. Immunol. 17, 30-48.

Wright, M. D., Moseley, G. W. and van Spriel, A. B. (2004). Tetraspanin microdomains in immune cell signalling and malignant disease. Tissue Antigens 64, 533-542.

Xu, S., Huo, J., Gunawan, M., Su, I.-H. and Lam, K.-P. (2009). Activated dectin-1 localizes to lipid raft microdomains for signaling and activation of phagocytosis and cytokine production in dendritic cells. J. Biol. Chem. 284, 22005-22011.

Yan, J., Wu, B., Huang, B., Huang, S., Jiang, S. and Lu, F. (2014). Dectin-1-CD37 association regulates IL-6 expression during Toxoplasma gondii infection. Parasitol. Res. 113, 2851-2860.

Zeiler, M., Moser, M. and Mann, M. (2014). Copy number analysis of the murine platelet proteome spanning the complete abundance range. Mol. Cell. Proteomics 13, 3435-3445.

Zhang, J., Somani, A.-K. and Siminovitch, K. A. (2000). Roles of the SHP-1 tyrosine phosphatase in the negative regulation of cell signalling. Semin. Immunol. 12, 361-378.
Zhang, X. A., Bontrager, A. L. and Hemler, M. E. (2001). Transmembrane-4 superfamily proteins associate with activated protein kinase $C(P K C)$ and link PKC to specific beta(1) integrins. J. Biol. Chem. 276, 25005-25013.

Zimmerman, B., McMillan, B., Seegar, T., Kruse, A. and Blacklow, S. (2016). Crystal structure of human tetraspanin CD81 reveals a conserved intramembrane binding cavity. FASEB J. $\mathbf{3 0}$, lb71.

Zuidscherwoude, M., de Winde, C. M., Cambi, A. and van Spriel, A. B. (2014). Microdomains in the membrane landscape shape antigen-presenting cell function. J. Leukoc. Biol. 95, 251-263.

Zuidscherwoude, M., Göttfert, F., Dunlock, V. M. E., Figdor, C. G., van den Bogaart, G. and van Spriel, A. B. (2015). The tetraspanin web revisited by superresolution microscopy. Sci. Rep. 5, 12201.

Zuidscherwoude, M., Worah, K., van der Schaaf, A., Buschow, S. I. and van Spriel, A. B. (2017a). Differential expression of tetraspanin superfamily members in dendritic cell subsets. PLoS ONE 12, e0184317.

Zuidscherwoude, M., Dunlock, V. M. E., van den Bogaart, G., van der Schaaf, A., van Oostrum, J., Goedhart, J., IntHout, J., Hämmerling, G. J., Tanaka, S. et al. (2017b). Tetraspanin microdomains control localized protein kinase C signaling in B cells. Sci. Signal. 10, eaag2755 
A

WT
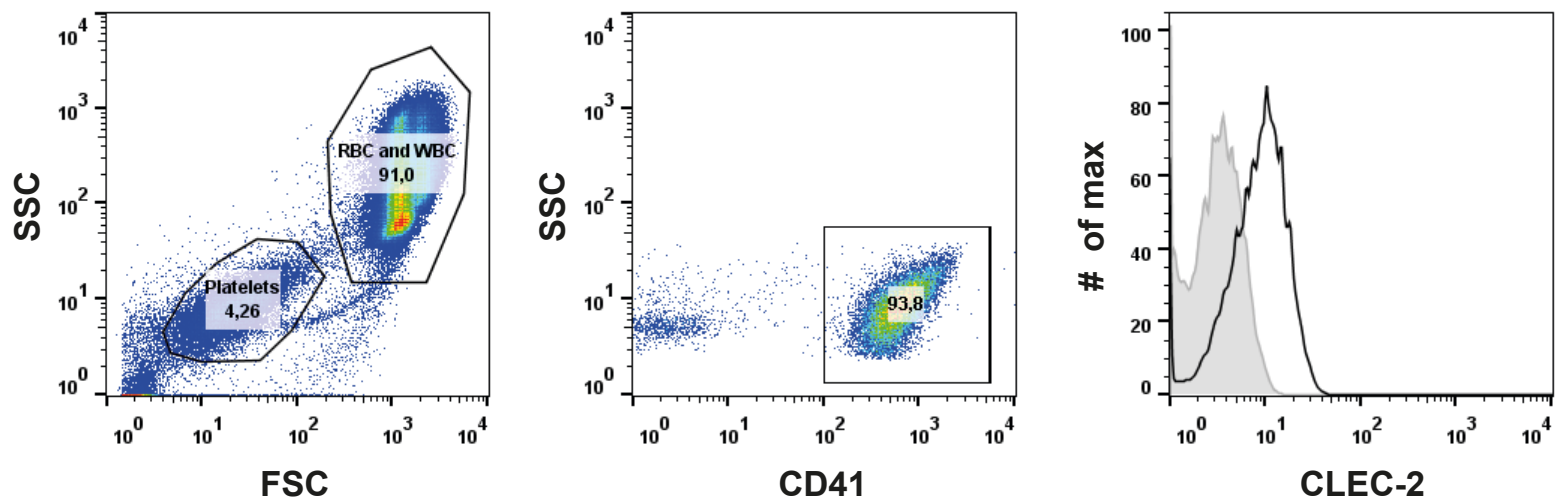

Cd37-/-
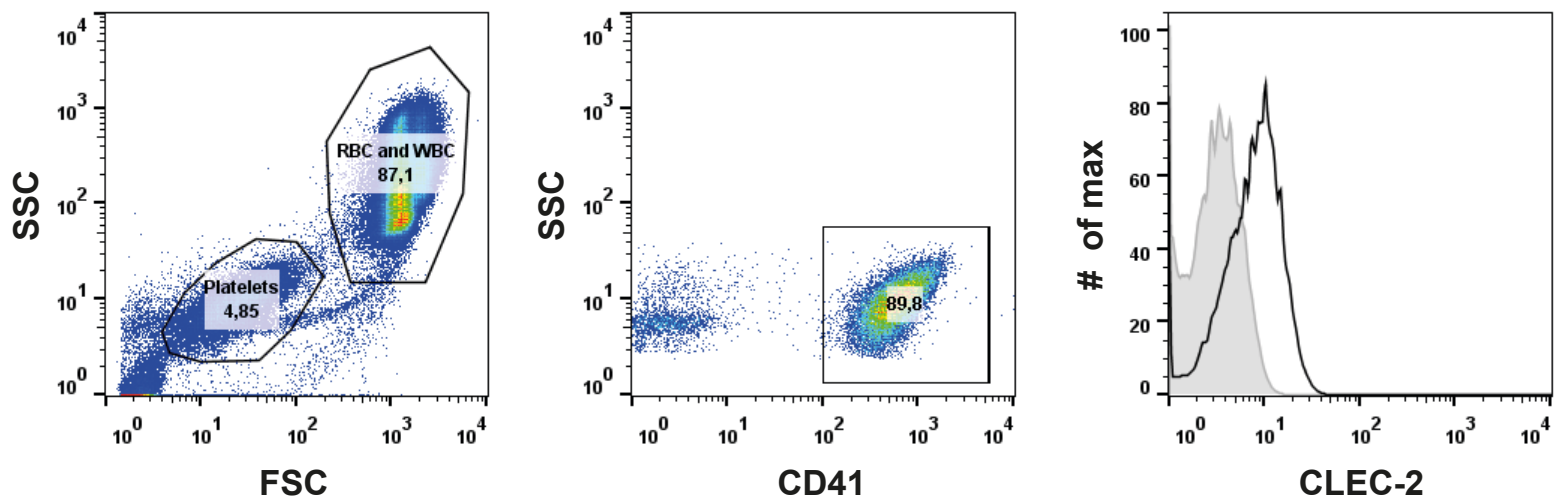

B

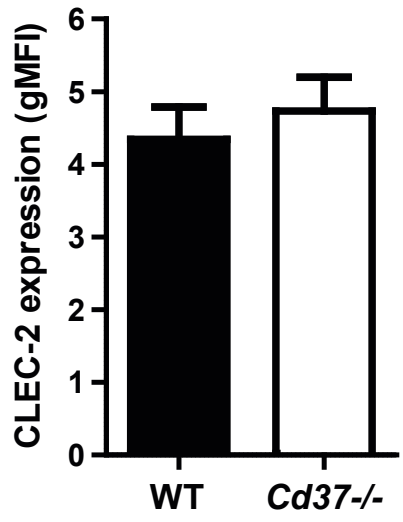

Figure S1. Cd37-/- platelets express normal CLEC-2 levels. (A) Flow cytometric analysis of CLEC-2 expression (right histogram, black line) on murine $\mathrm{CD} 41^{+}$blood platelets (middle histogram) from WT (upper panel) and Cd37-/- (lower panel) mice. $\mathrm{RBC}=$ red blood cells, $\mathrm{WBC}=$ white blood cells. (B) Quantification of flow cytometric analysis of CLEC-2 expression on WT (white bar) and Cd37-/- (black bar) $\mathrm{CD} 41^{+}$platelets. Values are corrected for isotype control. gMFI = geometric mean fluorescence intensity. Data are shown as mean + s.e.m. from $n=6$ mice per genotype. 

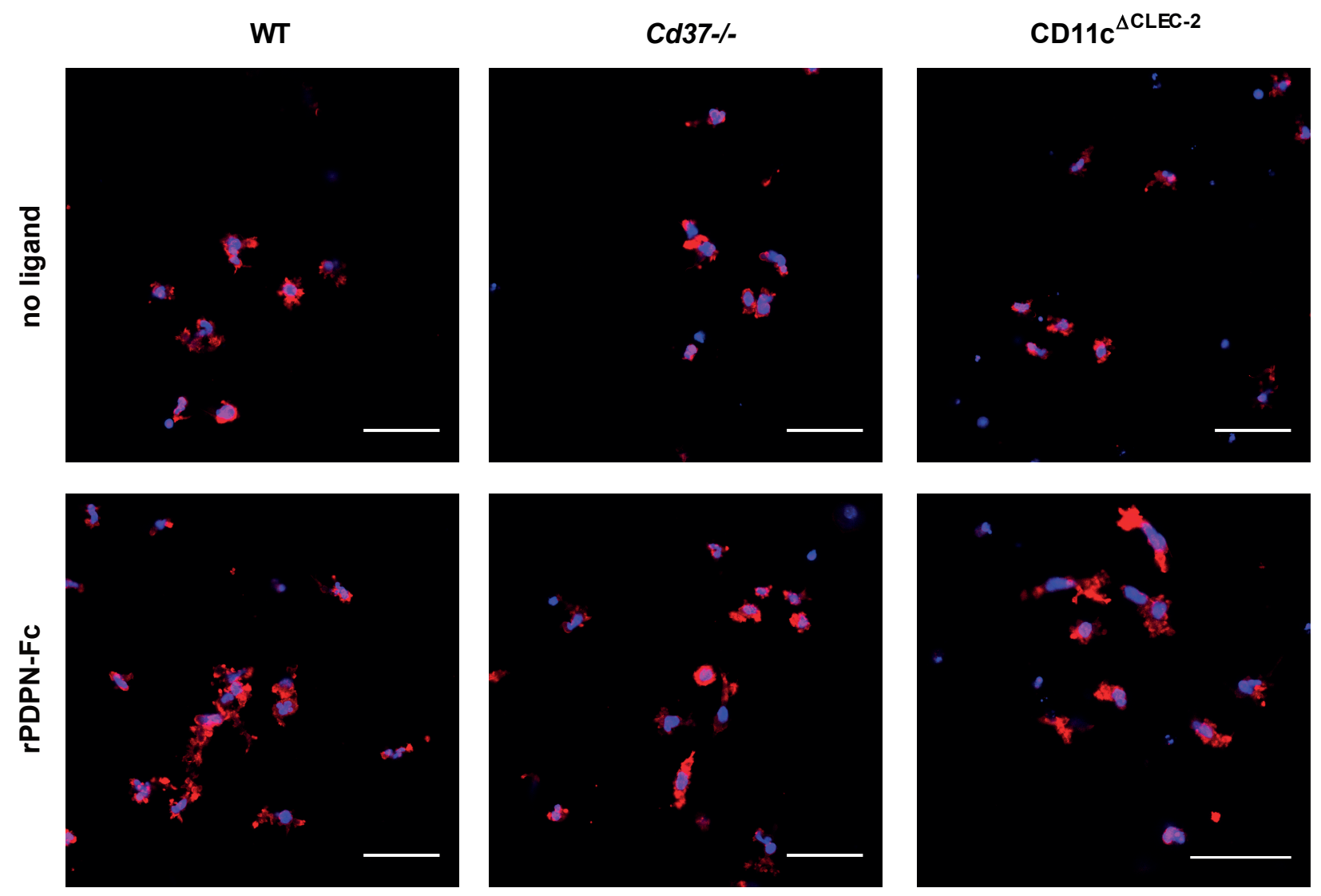

Figure S2. CD37 controls formation of actin protrusions by DCs in response to podoplanin. WT (left), Cd37-/- (middle) or CD11 $\mathrm{c}^{\triangle \mathrm{CLEC}-2}$ (right) DCs were stimulated in a 3D collagen gel with (bottom row) or without (upper row) recombinant podoplanin-Fc (rPDPN-Fc). Cells were stained for F-actin (red) and nucleus (blue) and imaged with a Leica SP5 confocal fluorescence microscope. One representative image is shown for each condition. Scale bar $=50 \mu \mathrm{m}$. 
A

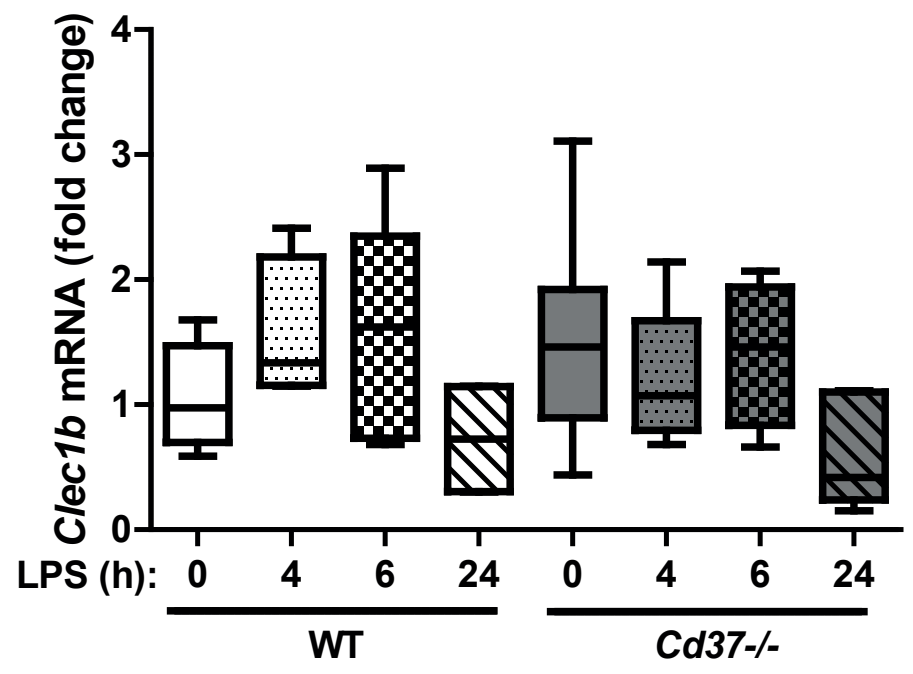

B

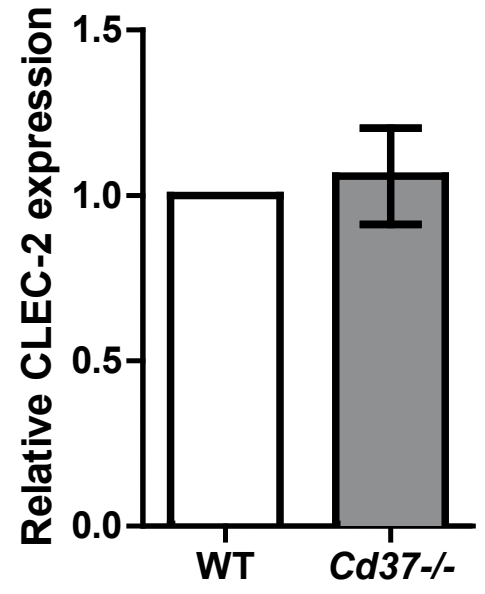

Figure S3. No difference in CLEC-2 mRNA and membrane protein expression between WT and Cd37-/bone marrow-derived DCs (BMDCs) upon LPS stimulation. (A) Clec1b mRNA expression in WT (white boxes) and Cd37-/- (grey boxes) BMDCs after indicated time points of LPS stimulation. Clec1b mRNA expression is calculated as fold change compared to unstimulated (Oh) WT BMDCs. Data shown as Tukey Box \& whiskers from 4-8 independent cultures per time point. (B) CLEC-2 membrane protein expression on WT (white bar) and Cd37-/- (grey bar) CD11c-positive BMDCs stimulated with $10 \mathrm{ng} / \mathrm{mL}$ LPS for 24 hours. Data was first normalized to an isotype control antibody, and secondly to CLEC-2 expression levels in WT BMDCs (set at 1). Data shown as mean \pm s.e.m of four independent experiments. 
A

TF:

mock

GFP-mCLEC-2

mCD37-mCherry +

GFP-mCLEC-2
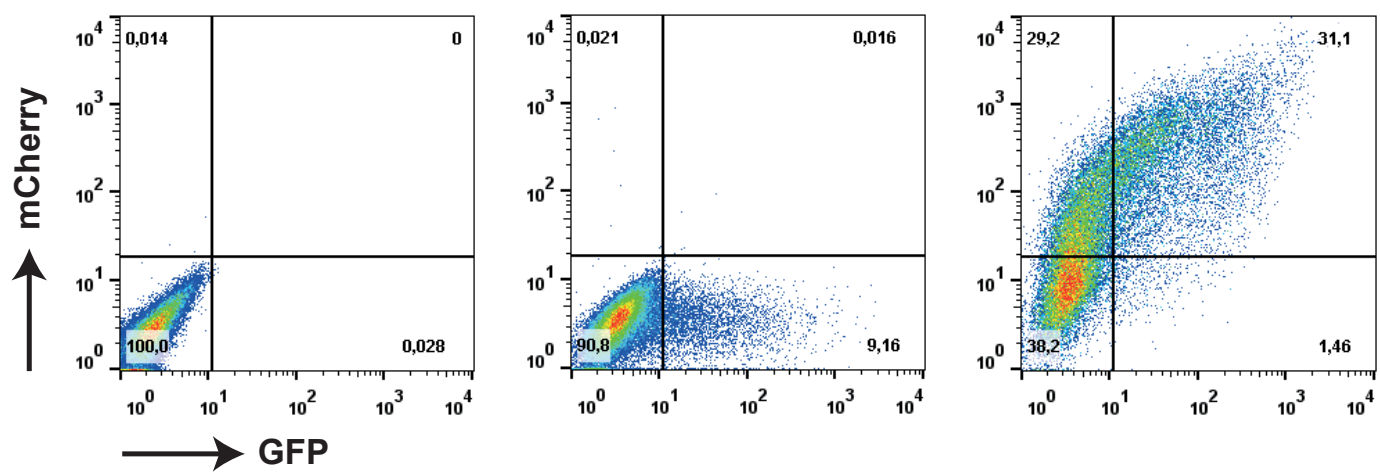

B
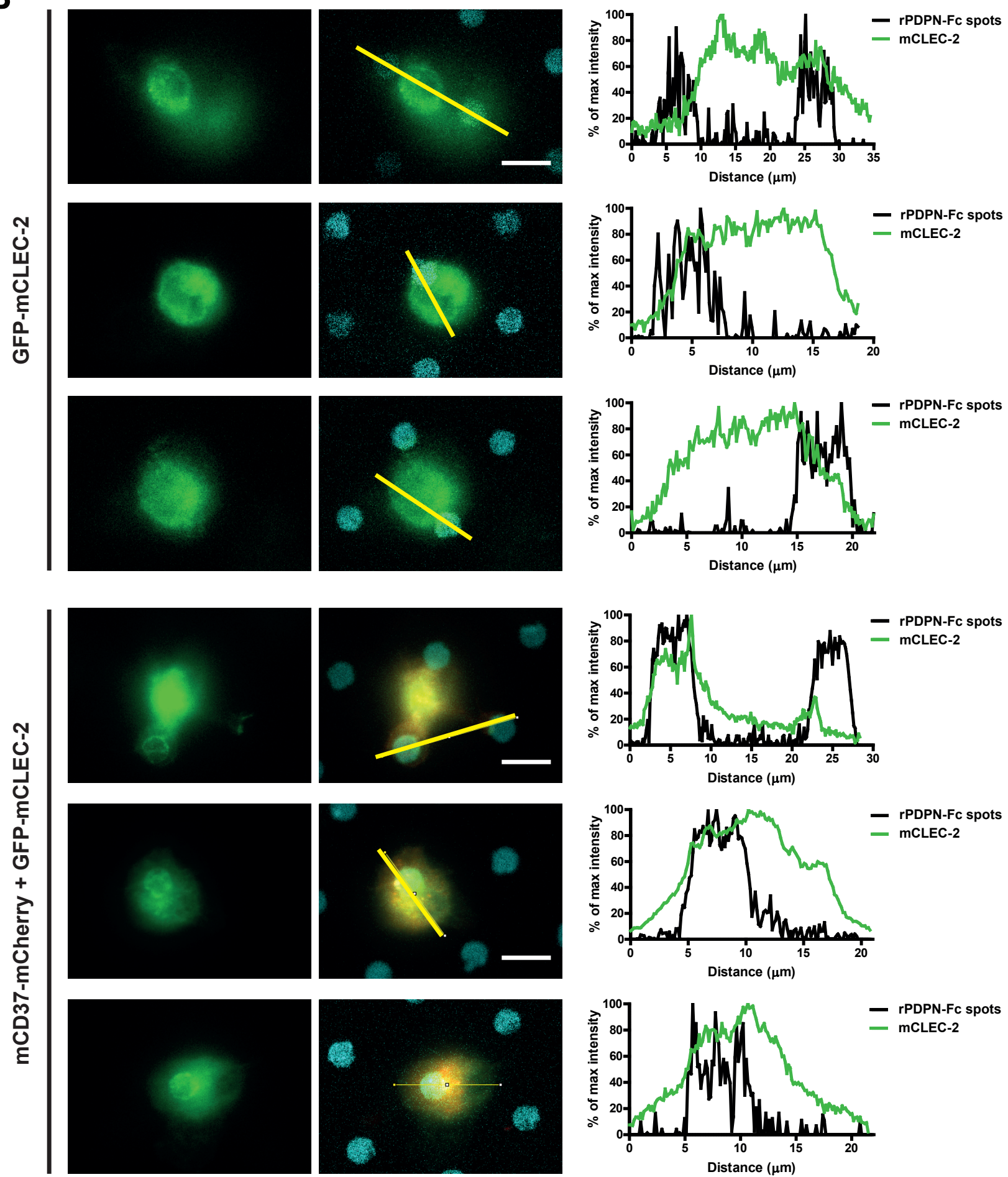
Figure S4. CLEC-2 recruitment to podoplanin is dependent on CD37. (A) Percentage of positive RAW macrophages after transfection with either GFP-mCLEC-2 alone (middle) or in combination with mCD37mCherry (right). Mock transfected cells (left) were used as negative control. TF = transfection condition . (B) RAW macrophages transfected with GFP-mCLEC-2 alone (upper three rows) or in combination with mCD37-mCherry (bottom three rows) were incubated on recombinant podoplanin-Fc (rPDPN-Fc) spots and analyzed after 12 min using an epi-fluorescence microscope. Left: Green = GFP, red = mCherry, blue = rPDPN-Fc spots. Two representative cells are shown per condition. Scale bar $=10 \mu \mathrm{m}$. Right: Graphs represent intensity profile of GFP-mCLEC-2 (green line) or rPDPN-Fc spot (black line) across the yellow line. 
A CD37 (clone Duno85)

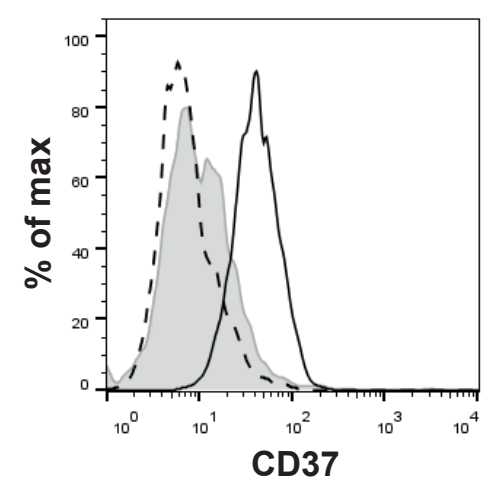

B podoplanin (clone 8.1.1)

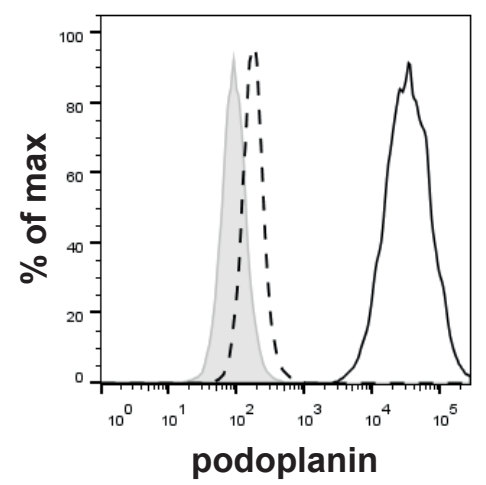

Figure S5. Antibody validation. (A) WT (black line) and Cd37-/- (dashed line) splenocytes were stained with rat anti-mouse CD37 (clone Duno85, Biolegend), or an isotype control (grey), followed by an appropriate secondary antibody. Results show CD37 staining on CD19+B220+ B cells. (B) Podoplaninexpressing (WT; black line) or podoplanin-knockout (using CRISPR; dashed line) FRCs were stained with hamster anti-mouse podoplanin (clone 8.1.1, Acris Antibodies), followed by an appropriate secondary antibody. Background signal (unstained WT FRCs) is shown in grey. 


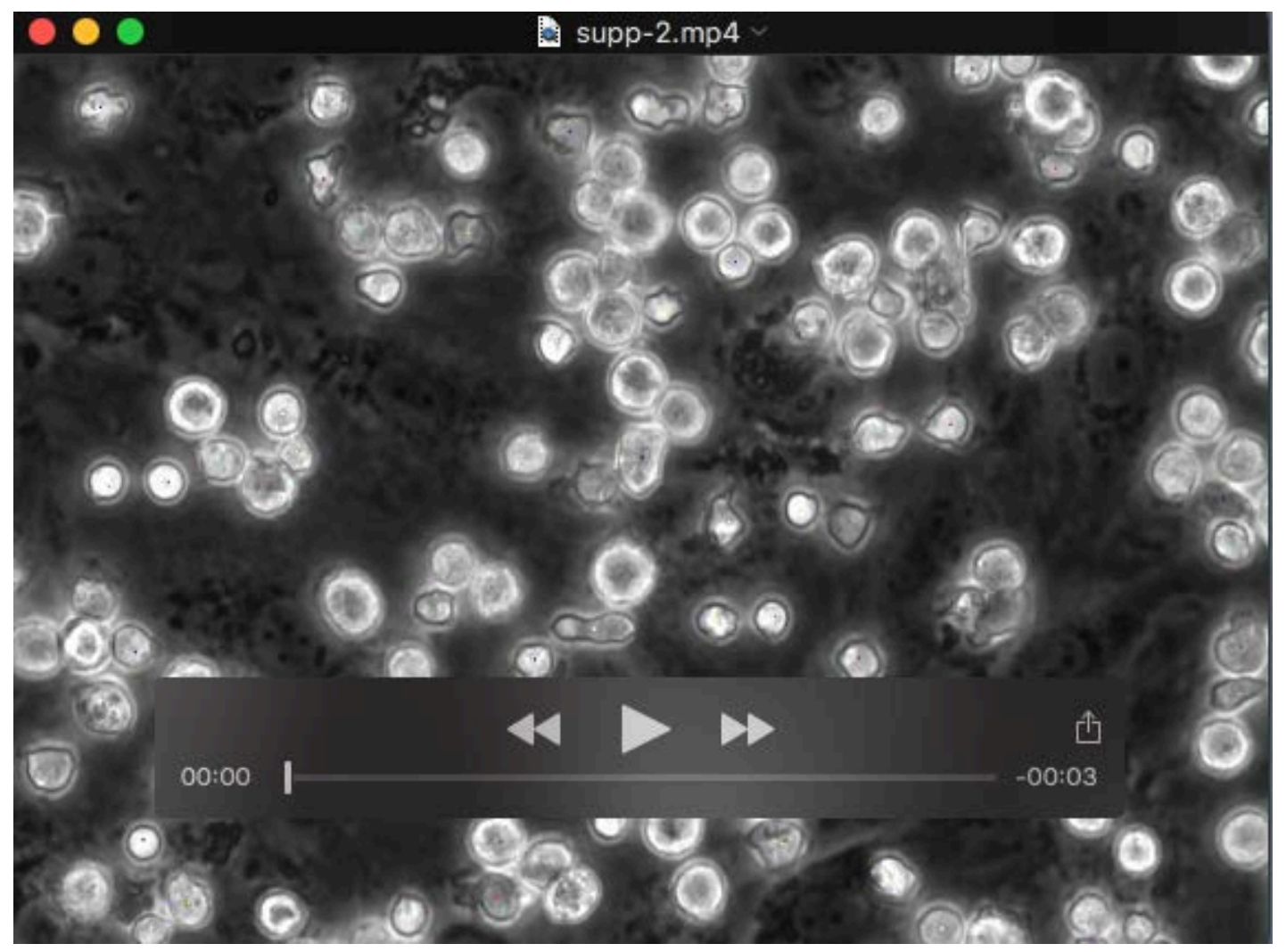

Movie 1. Migration of WT BMDCs on LECs. CLEC-2+ (LPS-stimulated) WT BMDCs were seeded on a TNF $\alpha-$ stimulated LEC monolayer. After $10 \mathrm{~min}$, non-adherent BMDCs were washed away and time-lapse imaging was performed for $5 \mathrm{~min}$ ( 1 image every $10 \mathrm{sec}$ ). Cell tracks of live cells were analyzed using the Manual Tracking plugin in Fiji/ImageJ software. 


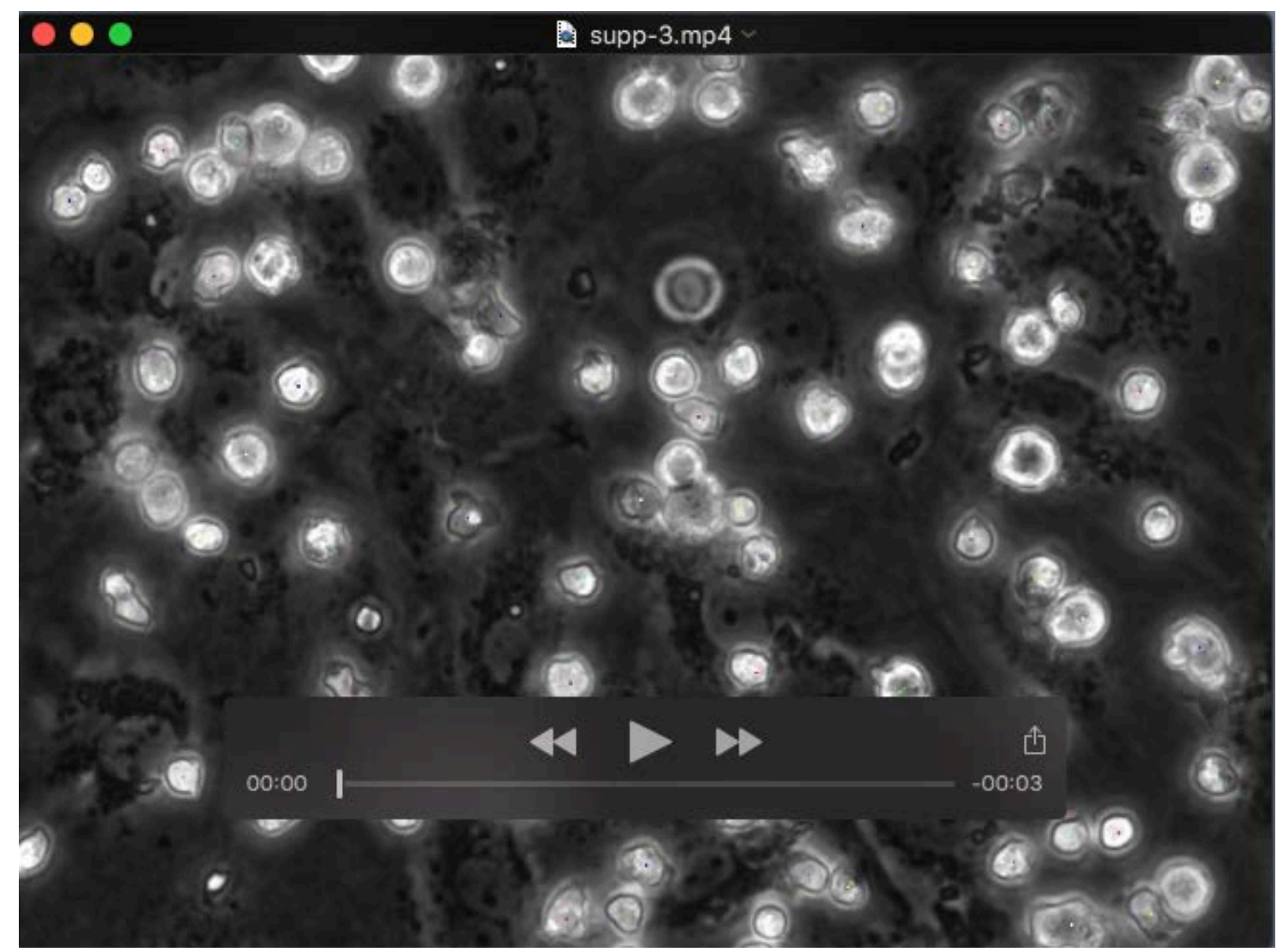

Movie 2. Migration of Cd37-/- BMDCs on LECs. CLEC-2+ (LPS-stimulated) Cd37-/- BMDCs were seeded on a TNF $\alpha$-stimulated LEC monolayer. After $10 \mathrm{~min}$, non-adherent BMDCs were washed away and timelapse imaging was performed for 5 min ( 1 image every 10sec). Cell tracks of live cells were analyzed using the Manual Tracking plugin in Fiji/ImageJ software. 